\title{
Kondo screening in Co adatoms with full Coulomb interaction
}

\author{
Angelo Valli $\odot,{ }^{1}$ Marc Philipp Bahlke, ${ }^{2}$ Alexander Kowalski $\odot,{ }^{3}$ Michael Karolak, ${ }^{4}$ Carmen Herrmann, ${ }^{2}$ \\ and Giorgio Sangiovanni (i) $^{4}$ \\ ${ }^{1}$ Institut für Theoretische Physik, Technische Universität Wien, Wiedner Hauptstrasse 8-10, 1040 Vienna, Austria \\ ${ }^{2}$ Institut für Anorganische und Angewandte Chemie, Universität Hamburg, Martin-Luther-King-Platz 6, 20146 Hamburg, Germany \\ ${ }^{3}$ Institut für Theoretische Physik und Astrophysik, Universität Würzburg, Am Hubland, 97074 Würzburg, Germany \\ ${ }^{4}$ Institut für Theoretische Physik und Astrophysik and Würzburg-Dresden Cluster of Excellence ct.qmat, Universität Würzburg, \\ 97074 Würzburg, Germany
}

(Received 14 April 2020; revised 19 August 2020; accepted 19 August 2020; published 17 September 2020)

\begin{abstract}
Using a numerically exact first-principles many-body approach, we revisit the "prototypical" Kondo case of a cobalt impurity on copper. Even though this is considered a well understood example of the Kondo effect, we reveal an unexpectedly strong dependence of the screening properties on the parametrization of the local Coulomb tensor. As a consequence, the Kondo temperature can vary by orders of magnitude depending on the complexity of the parametrization of the electron-electron interaction. Further, we challenge the established picture of a spin-1 moment involving two cobalt $d$ orbitals only, as orbital-mixing interaction terms boost the contribution of the remainder of the $d$ shell.
\end{abstract}

DOI: 10.1103/PhysRevResearch.2.033432

\section{INTRODUCTION}

The Kondo effect arises when a local magnetic moment is quantum mechanically screened by the conduction electrons of a metallic host. Explained by Kondo in the 1960s [1], this phenomenon has been extensively studied thereafter within Anderson's poor man's approach and Wilson's renormalization group [2]. As a direct consequence of the screening of the impurity magnetic moment, the spin susceptibility undergoes a crossover from a Curie-Weiss to a Pauli behavior upon lowering the temperature. At the same time, the Abrikosov-Suhl-Kondo resonance [2-4] emerges in the electronic spectral function at the Fermi level. Magnetic response functions and electron transport are therefore suitable probes of the Kondo effect. Despite its well-defined characterization, the signatures of the Kondo effect emerge at energy scales of the order of the Kondo temperature $T_{K}$, which is often of the order of a few kelvin, making the theoretical description of realistic Kondo systems intrinsically hard. Further, the Fermiliquid properties emerging below the Kondo temperature $T_{K}$ are typically reached via smooth crossovers rather than with sharp transitions, complicating also the experimental detection.

One case of Kondo effect considered to be simple and relatively well understood is that of a Co single impurity on a metallic substrate, such as $\mathrm{Cu}$ [5-8], $\mathrm{Au} \mathrm{[6],} \mathrm{and} \mathrm{Ag}$ [6,9]. In particular, scanning tunneling spectroscopy (STM)

Published by the American Physical Society under the terms of the Creative Commons Attribution 4.0 International license. Further distribution of this work must maintain attribution to the author(s) and the published article's title, journal citation, and DOI. has revealed how Co adatoms on $\mathrm{Cu}$ hosts display sharp peaks or Fano-like resonances at zero bias [5-8,10,11], which are commonly interpreted as a clear experimental signature of the Kondo screening, although the origin of these features has been recently challenged [12]. Experimental estimates of the Kondo scale yield, e.g., $T_{K} \approx 88 \mathrm{~K}$ and $54 \mathrm{~K}$ for Co on $\mathrm{Cu}(001)$ and $\mathrm{Cu}(111)$, respectively $[5,6]$.

However, even in the case of a single impurity, for transition metal adatoms the theoretical description of the Kondo effect is difficult, since the whole $d$ shell is likely to play a role in the screening. So far, the theoretical understanding of single $\mathrm{Co}$ impurities on $\mathrm{Cu}$ [13-16] stresses the main role played by two of the five Co- $d$ orbitals. In the case of $\mathrm{Co} / \mathrm{Cu}(001)$ - on which we shall focus below-the $d_{x y}$ and $d_{z^{2}}$ orbitals are Kondo active, in the sense that they are half-filled and carry a magnetic moment. Due to the different symmetry, for $\mathrm{Co} / \mathrm{Cu}(111)$ the $d_{z^{2}}$ orbital is instead fully occupied, and the magnetic moment arises from one of the two doublets with $E$ symmetry $[13,16]$. In general, different crystalline environments determine variations in the local electronic structure of the impurity and lead to drastically different Kondo resonance line shapes observed in STM experiments $[8,13]$. Furthermore, the many-body nature of the Kondo effects manifests itself also in a strong dependence of $T_{K}$ on the occupation of the Co $3 d$ shell $[6,8,11,14]$. This also means that the hybridization and the charge transfer between the impurity and the substrate play an important role. This is reflected in a strong dependence of $T_{K}$ on, e.g., the adatom adsorption distance [16,17], in agreement with the experiments [7,8]. In general, the Kondo scale depends exponentially on the parameters of the theoretical model, making reliable estimates of $T_{K}$ extremely hard.

For the same reason, it is also difficult to exactly pinpoint the details of the physical processes underlying the Kondo 
screening in these systems. Interestingly, theoretical calculations indicate that the spin state of Co is $S=1$ on both the $\mathrm{Cu}(001)$ and $\mathrm{Cu}(111)$ surfaces [16]. However, evidence for very different Kondo scales for the Kondo-active orbitals of $\mathrm{Co} / \mathrm{Cu}(001)[14,16]$ suggest an underscreened (or possibly a two-stage [18]) Kondo effect to take place, while a single $T_{K}$ is expected for the magnetic doublet of $\mathrm{Co} / \mathrm{Cu}(111)$, although the degeneracy could be lifted by, e.g., spin-orbit coupling [16]. On the other hand, Nevidomskyy and Coleman [19] showed that, in the case of a multiorbital impurity, the stabilization of an impurity high-spin state due to Hund's coupling leads to a strong reduction of the Kondo coupling, and consequently of $T_{K}$, with respect to the spin $S=1 / 2$ case [20,21]. Robust numerical evidence that the Nevidomskyy-Coleman scenario is realized in idealized model systems comes, e.g., from Ref. [22]. This seems, however, at odds with the relatively high estimates of $T_{K}$ for these systems emerging from experiments [5-7]. Hence the question is whether or not, or under which conditions, the Kondo screening of $\mathrm{Co}$ on a $\mathrm{Cu}$ substrate can be described this way upon cooling.

We identify two key players which may affect the mechanism of the Kondo screening, i.e., multiorbital correlation effects arising from the full treatment of the Co $3 d$ shell, rather than restricting the description to the Kondo-active orbitals only, and the approximation of the form of the Coulomb interaction. Using a combination of density functional theory (DFT) and numerically exact quantum Monte Carlo (QMC) we analyze the many-body processes leading to the formation and the screening of the local moment on a Co impurity on $\mathrm{Cu}(001)$ in its full realistic complexity. We provide a comparative analysis of the role of the parametrization of the Coulomb interaction, which is so-far scarcely investigated in a systematic way. In particular, we take into account the full Coulomb tensor in the whole Co $3 d$ multiplet, hitherto either simplified $[14,16]$ or included only at high temperatures $[11,15,23]$, and we push our calculations down to temperatures which are relevant to the Kondo screening.

The paper is organized as follows. In Sec. II we provide the details of the $a b$ initio and many-body calculations for $\mathrm{Co} / \mathrm{Cu}(001)$. In Sec. III we discuss the possible Kondo scenarios, and in Sec. IV we analyze the screening properties, providing evidence which supports the important role played by the approximations of the Coulomb tensor. Finally, Sec. V contains a discussion of our results in light of previous studies in the literature, as well as our conclusions.
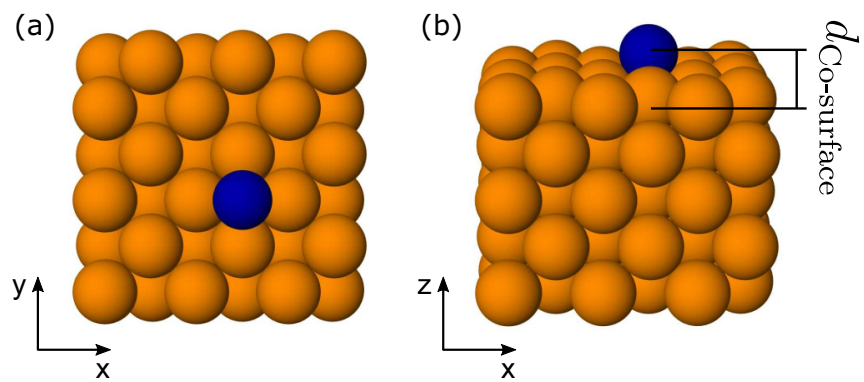

FIG. 1. Top (a) and side (b) view of the $\mathrm{Co} / \mathrm{Cu}(001)$ unit cell as used in this study. The adsorption distance between the Co adatom and the substrate is set to $d_{\text {Co-surface }}=1.52 \AA$ [17].

\section{METHODOLOGY}

\section{A. DFT ++}

Here we investigate the correlation effects of a single Co adatom on a $\mathrm{Cu}(001)$ surface using a combination of DFT within the local density approximation (LDA) and the numerical solution of an Anderson impurity model (AIM) with realistic parameters. This approach is commonly referred to as DFT ++ in the literature [24,25].

The DFT calculations have been performed with the Vienna $a b$ initio simulation program (VASP) [26,27] using the projector augmented plane wave (PAW) basis set. We modeled the $\mathrm{Cu}(001)$ surface as a $4 \times 4$ slab consisting of five $\mathrm{Cu}$ layers using the experimental [28] lattice constant of 3.615 $\AA$. The Co adatom was placed in the fourfold-hollow position (see Fig. 1) at an adsorption distance $d_{\text {Co-surface }}=1.52 \AA$ with respect to the first $\mathrm{Cu}(001)$ layer, which we identified in one of our earlier works [17] to be the energetically favored distance, in agreement with previous literature [13]. We used a $k$ mesh centered around the $\Gamma$ point of size $100 \times 100 \times 1 k$ points in order to achieve a sufficiently accurate description of our $\mathrm{Cu}(001)$ substrate. This will be necessary for the parametrization of the AIM, especially at low temperatures (this important aspect is discussed in Appendix A in more detail).

With the combination of DFT and an AIM, we can take into account the correlation effects on the Co atom explicitly as well as the realistic complexity of its hybridization with the $\mathrm{Cu}$ substrate. The Hamiltonian of the AIM reads

$$
\hat{H}=\sum_{\nu \sigma} \epsilon_{v} \hat{c}_{\nu \sigma}^{\dagger} \hat{c}_{\nu \sigma}+\sum_{v i \sigma}\left(V_{\nu i} \hat{c}_{\nu \sigma}^{\dagger} \hat{d}_{i \sigma}+V_{v i}^{*} \hat{d}_{i \sigma}^{\dagger} \hat{c}_{\nu \sigma}\right)+\sum_{i \sigma} \epsilon_{i} \hat{d}_{i \sigma}^{\dagger} \hat{d}_{i \sigma}+\frac{1}{2} \sum_{i j k l} \sum_{\sigma \sigma^{\prime}} U_{i j k l} \hat{d}_{i \sigma}^{\dagger} \hat{d}_{j \sigma^{\prime}}^{\dagger} \hat{d}_{l \sigma^{\prime}} \hat{d}_{k \sigma},
$$

where $\hat{c}_{\nu \sigma}^{\dagger}\left(\hat{c}_{\nu \sigma}\right)$ denotes the creation (annihilation) operators for an electron with spin $\sigma$ in the $\nu$ th bath state (in this work, the $\mathrm{Cu}$ surface) with energy $\epsilon_{\nu}$, whereas $\hat{d}_{i \sigma}^{\dagger}\left(\hat{d}_{i \sigma}\right)$ denotes the corresponding operators for the $i$ th localized $3 d$ orbital of the impurity (in this work, the Co $3 d$ shell) with energy $\epsilon_{i}$. The bath and impurity electrons are coupled via the hybridization $V_{v i}$. For QMC techniques, it is convenient to reformulate the AIM (1) in the action formalism, and integrate out the degrees of freedom of the bath to obtain a retarded hybridization function

$$
\Delta_{i}(\omega)=\sum_{\nu} \frac{V_{\nu i} V_{v i}^{*}}{\omega+\imath 0^{+}-\epsilon_{i}},
$$

which effectively embeds the impurity into the substrate. Our results, shown in Fig. 2, are compatible with others found in 


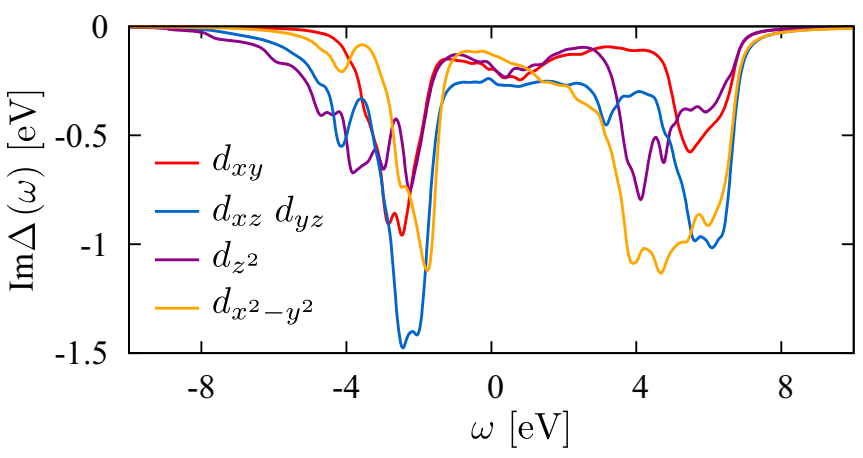

FIG. 2. Orbital-resolved hybridization function $\operatorname{Im} \Delta(\omega)$ for $\mathrm{Co} / \mathrm{Cu}(001)$. The corresponding values at the Fermi level, $\Gamma=$ $-\operatorname{Im} \Delta(0)$ for each orbital, are reported in Table I.

the literature [14]. The hybridization function is then transformed into the Matsubara representation $\Delta_{i}(\omega) \rightarrow \Delta_{i}\left(\imath \omega_{n}\right)$ for QMC sampling. The values of the orbital-dependent effective crystal fields $\epsilon_{i}+\operatorname{Re} \Delta_{i}(\infty)$ and hybridization to the substrate $\Gamma_{i}=-\operatorname{Im} \Delta_{i}(0)$ are given in Table I for reference.

Finally, the tensor

$$
U_{i j k l}=\int d r d r^{\prime} \psi_{i}^{*}(r) \psi_{j}^{*}\left(r^{\prime}\right) \frac{e^{2}}{\left|r-r^{\prime}\right|} \psi_{k}(r) \psi_{l}\left(r^{\prime}\right)
$$

describes the local Coulomb interaction (we dropped the spin indices, for simplicity) as introduced by Slater [29], with $\psi_{\alpha}(\alpha=i, j, k, l)$ being in general any atom-centered basis function and $e^{2} \frac{1}{\left|r-r^{\prime}\right|}$ the long-range Coulomb potential.

\section{B. Coulomb tensor}

The last term of Eq. (1) describes the local interaction within the impurity $3 d$ shell. The full Coulomb interaction $U_{i j k l}$ is in general a four-index tensor, which, in the language of second quantization, corresponds to different combinations of the creation and annihilation operators of the two-body interaction. However, due to the extreme numerical complexity required to take into account all possible four-fermion terms, it is common practice so far to consider approximate interaction schemes. Therefore, most previously published results have been obtained neglecting-in a nonsystematic

TABLE I. Single-particle DFT parameters defining the AIM for $\mathrm{Co} / \mathrm{Cu}(001)$, i.e., the effective crystal field $\epsilon_{i}+\operatorname{Re} \Delta_{i}(\infty)$ and the coupling to the substrate $\Gamma_{i}=-\operatorname{Im} \Delta_{i}(0)$ for each orbital in the Co $3 d$ shell. The $C_{4 v}$ point-group symmetry is enforced at the DFT level, so that $d_{x z}$ and $d_{y z}$ are degenerate. In addition to the crystal field, we also include a double counting correction $\mu_{\mathrm{DC}}=28.0 \mathrm{eV}$ to constrain the occupation of the $3 d$ shell to $n_{d}=8.0$ electrons.

\begin{tabular}{lcc}
\hline \hline Co $3 d$ orbital & $\epsilon_{i}+\operatorname{Re} \Delta_{i}(\infty)(\mathrm{eV})$ & $\Gamma_{i}(\mathrm{eV})$ \\
\hline$d_{x y}$ & -0.226 & 0.196 \\
$d_{x z}$ & -0.403 & 0.244 \\
$d_{z^{2}}$ & -0.295 & 0.180 \\
$d_{y z}$ & -0.403 & 0.244 \\
$d_{x^{2}-y^{2}}$ & -0.221 & 0.128 \\
\hline \hline
\end{tabular}

and uncontrolled way-parts of the Coulomb tensor. With the advent of continuous-time quantum Monte Carlo (CT-QMC) methods (see Ref. [30] for a review), it has become possible to treat the full Coulomb interaction without approximations. There are already indications in the literature [23] that the structure of the full Coulomb interaction is important to describe the physics of $\mathrm{Co} / \mathrm{Cu}(001)$. We will show that different parametrizations of the Coulomb interaction also give rise to substantially dissimilar Kondo screening properties.

Below we describe the properties of $U_{i j k l}$ in different approximation schemes. Within the simplest parametrization, one retains only the "density-density" terms, i.e., those in which the four operators are contracted in pairs of number operators $\hat{n}_{i \sigma}=\hat{d}_{i \sigma}^{\dagger} \hat{d}_{i \sigma}$. Within the density-density approximation, the Coulomb tensor in Eq. (1) reduces to

$$
\hat{H}_{\mathrm{D}}=\sum_{i} U_{i i} \hat{n}_{i \uparrow} \hat{n}_{i \downarrow}+\sum_{i \neq j} \sum_{\sigma \sigma^{\prime}}\left(U_{i j}-J_{i j} \delta_{\sigma \sigma^{\prime}}\right) \hat{n}_{i \sigma} \hat{n}_{j \sigma^{\prime}} .
$$

In terms of the Coulomb tensor, the above parameters $U_{i i}=$ $U_{i i i i}$ and $U_{i j}=U_{i j i j}$ denote the intra- and interorbital (direct) interactions, while $J_{i j}=U_{i j j i}$ denotes the density-density Hund's exchange coupling for $\sigma=\sigma^{\prime}$ (see Appendix B for all definitions and symmetry relations).

Including also the missing two-body scattering terms, which describe "spin-flip" $\left(J_{i j}=U_{i j j i}\right.$ for $\left.\sigma \neq \sigma^{\prime}\right)$ and "pairhopping" $\left(J_{i j}=U_{i i j j}\right.$ for $\left.\sigma \neq \sigma^{\prime}\right)$ processes between electrons on different orbitals, gives rise to the so-called "Kanamori" parametrization, of the form

$$
\hat{H}_{\mathrm{K}}=\hat{H}_{\mathrm{D}}+\sum_{i \neq j} J_{i j}\left(\hat{d}_{i \uparrow}^{\dagger} \hat{d}_{j \downarrow}^{\dagger} \hat{d}_{i \downarrow} \hat{d}_{j \uparrow}-\hat{d}_{i \uparrow}^{\dagger} \hat{d}_{i \downarrow}^{\dagger} \hat{d}_{j \uparrow} \hat{d}_{j \downarrow}\right),
$$

which has the important consequence of restoring the rotational invariance of the Coulomb interaction. Finally, the "full Coulomb" interaction, given by the generic form

$$
\hat{H}_{\mathrm{C}}=\frac{1}{2} \sum_{i j k l} \sum_{\sigma \sigma^{\prime}} U_{i j k l} \hat{d}_{i \sigma}^{\dagger} \hat{d}_{j \sigma^{\prime}}^{\dagger} \hat{d}_{l \sigma^{\prime}} \hat{d}_{k \sigma},
$$

contains all possible terms allowed on the $3 d$ shell, without restrictions. In the case of a spherically symmetric atom, these terms can be described in terms of the Slater radial integrals [29,31] $F^{0}, F^{2}$, and $F^{4}$. With a spherically symmetric Coulomb tensor, one has the advantage of excluding sources of differences associated with specificities of the $\mathrm{Cu}(001)$ substrate, at the same time allowing us to reduce the number of interaction parameters to two: $U=F^{0}$ and $J=\frac{1}{14}\left(F^{2}+F^{4}\right)$. For instance, the intraorbital Hubbard repulsion becomes independent of the orbital index $i$ and is given by the relation $U_{i i}=F^{0}+\frac{8}{7} \frac{1}{14}\left(F^{2}+F^{4}\right)$. The different angular dependence of the five $d$ orbitals results in four different Hund's couplings $J_{i j}$, which can all be expressed in terms of $F^{2}$ and $F^{4}$, so that $U_{i j}=\left(U_{i i}+U_{j j}\right) / 2-2 J_{i j}$ (see, e.g., Refs. [32,33] and Appendix B for a detailed discussion).

\section{Details of the $\mathrm{Co} / \mathrm{Cu}(001)$ calculations}

We solve the AIM (1) by using the numerically exact CT-QMC method as implemented in the W2DYNAMICS package $[34,35]$. With the choices of interaction parameters $U=$ 
$4.0 \mathrm{eV}, J=0.9 \mathrm{eV}$, and ratio $F^{4} / F^{2}=0.625$, which completely determine the Coulomb tensor, the values we use in this study are very similar (although spherically symmetric) to those calculated for $\mathrm{Co} / \mathrm{Cu}(001)$ from first principles by Jacob within the constrained random phase approximation [14]. For the purpose of showing how significant the differences between the results obtained within various interaction schemes can be, we compare the magnetic properties of $\mathrm{Co} / \mathrm{Cu}(001)$ obtained by solving the impurity problem with the Coulomb interaction $\hat{H}_{\mathrm{C}}$ of Eq. (B1), as well as with its density-density and Kanamori approximations of Eqs. (4) and (5), respectively. We will show that different approximations of the Coulomb tensor lead to different physical pictures. In particular, the lowest temperature reached here for $\mathrm{Co} / \mathrm{Cu}(001)$ in the scope of the full Coulomb interaction is $T \simeq 33 \mathrm{~K}$, which is below the experimental estimates of $T_{K}$ for this system.

When interfacing many-body and $a b$ initio calculations, as within the DFT ++ scheme, one should also be aware of the so-called double-counting problem, which one encounters because part of the correlation energy (in this case on the Co $3 d$ shell) is already taken into account within DFT. Usually, one approximates the double-counting value from the fully localized limit (FLL) [36] or the around mean-field (AMF) [37] methods. Here we follow an alternative procedure, and choose the double counting in order to fix the Co $3 d$ occupation to $n_{d}=\sum_{i \sigma} n_{i \sigma}=8$ electrons, instead. One reason behind this choice is that the system has been investigated in several theoretical studies in an STM-like setup [14,16,38], where it is assumed that $\mathrm{Co}$ on $\mathrm{Cu}(001)$ has an $S=1$ spin state with an overall Co $3 d$ occupation of roughly $n_{d}=8$ electrons. This was also confirmed by correlated wave-function-based calculations, where a $\mathrm{Co} / \mathrm{Cu}_{n}$ cluster is embedded in a periodic potential [13]. Under the effect of the substrate crystal field, the Co $d_{x^{2}-y^{2}}$ and the $\left(d_{x z}, d_{y z}\right)$ doublet are completely full while the $d_{x y}$ and $d_{z^{2}}$ orbitals are both half filled. In this situation, $S=1$ high-spin configurations are expected to be locally dominant, which calls for a systematic analysis of the role of the Hund's coupling within the different approximations of the Coulomb tensor. However, we will also discuss deviations from integer filling of the Co $3 d$ shell, as they are expected to influence the screening properties of $\mathrm{Co} / \mathrm{Cu}(001)$ [14].

\section{POSSIBLE KONDO SCENARIOS}

The goal of this section is to determine how the Kondo screening mechanism can be influenced by the parametrization of the local Coulomb repulsion on the Co impurity. To this end, we are going to analyze in particular the finitetemperature spin and charge response functions, calculated at the Co site. We compare the three interaction schemes discussed in Sec. II B (i.e., density-density, Kanamori, and full Coulomb), especially focusing on the Co $d_{x y}$ and $d_{z^{2}}$ orbitals, which are identified as the Kondo-active orbitals in the literature $[14,16]$ (note the different orientation of the $x y$ plane here compared with these works). However, we will claim that more realistic descriptions of the Coulomb tensor favor a scenario in which also the other $3 d$ orbitals play an important role in the screening of the Co local moment.
The scheme presented in Fig. 3 anticipates the main results of the present paper. The electronic configuration of the Co $3 d$ shell hybridized with the $\mathrm{Cu}(001)$ surface is shown in Fig. 3(a). In the Co adatom with $n_{d}=8.0$ electrons in the $3 d$ shell, a high-spin state is always realized for temperatures above the Kondo regime. We find a link between the form of the Coulomb interaction, which strongly affects spin and charge fluctuations, as represented schematically in Fig. 3(b), and the possible mechanism behind the screening of the Co spin, indicated in Fig. 3(c). In the simplest approximation scheme, i.e., the one of Eq. (4), in which only the densitydensity part of the local Coulomb interaction is taken into account, the $\left(d_{x z}, d_{y z}, d_{x^{2}-y^{2}}\right)$ subspace is almost completely filled, and can be considered inert. Due to the strong Hund's coupling within the Kondo-active subspace, the Co impurity is locked into an $S=1$ state down to a few $\mathrm{K}$, when it can eventually be screened by the conduction electrons of the $\mathrm{Cu}$ surface, thus realizing the Nevidomskyy-Coleman scenario of the suppression of $T_{K}$ for an $S=1$ Kondo impurity. As we increase the complexity of the Coulomb tensor, by including interaction terms beyond the density-density approximation in the Kanamori parametrization of Eq. (5) and in the full Coulomb parametrizations of Eq. (B1), the most important effect that we observe is a progressive breakdown of the $\left(d_{x y}, d_{z^{2}}\right) S=1$ high-spin state. We can rationalize this effect in terms of two key players: (i) the enhancement of charge fluctuations within the whole Co $3 d$ multiplet and (ii) the frustration of the spin correlations due to the competition between all generalized exchange interactions in the Coulomb tensor, e.g., of the form $U_{i j j k}$ This includes Hund's coupling and the spin-flip processes $(i=k)$, as well as additional processes beyond the density-density approximation $(i \neq k)$, or of the form $U_{i j k l}$, with four different orbital indices. A thorough discussion of these terms is provided in Appendix B.

It is interesting to speculate on the suitable screening mechanisms which could replace the Nevidomskyy-Coleman scenario for $\mathrm{Co} / \mathrm{Cu}$ systems, in order to look for their characteristics in our numerical analysis. One possibility is the underscreened Kondo effect, where the Co spin is only partially screened by the substrate. Depending on how many modes of the host effectively couple to the impurity, a Noziéres Fermi liquid can be recovered at lower $T$ by screening the remaining spin (thus realizing a two-stage Kondo effect). In the regime where the charge fluctuations become dominant, the $d_{x y}$ and $d_{z^{2}}$ orbitals may also behave as a pair of $S=1 / 2$ replicas, which are screened at possibly very different Kondo temperatures. Moreover, depending on the degree of orbital degeneracy of the $3 d$ multiplet, an SU(4) Kondo effect could also take place. The latter may be relevant for the $\mathrm{Co} / \mathrm{Cu}(111)$ case, where the Co magnetic state is actually a doublet [16]. The increased symmetry, from an SU(2) spinKondo to an SU(4) spin-orbital Kondo-or even an SU(N) symmetry, involving also the rest of the $3 d$ multiplet-is generally expected to result in a single enhanced Kondo scale [39]. All the above mechanisms would be compatible with the relatively high $T_{K} \sim 50-100 \mathrm{~K}$ estimated by transport experiments [5-7].

While the general role of the Coulomb interaction emerges clearly from our calculations, a precise estimate of $T_{K}$ and the identification of the Kondo mechanism responsible for 
(a)

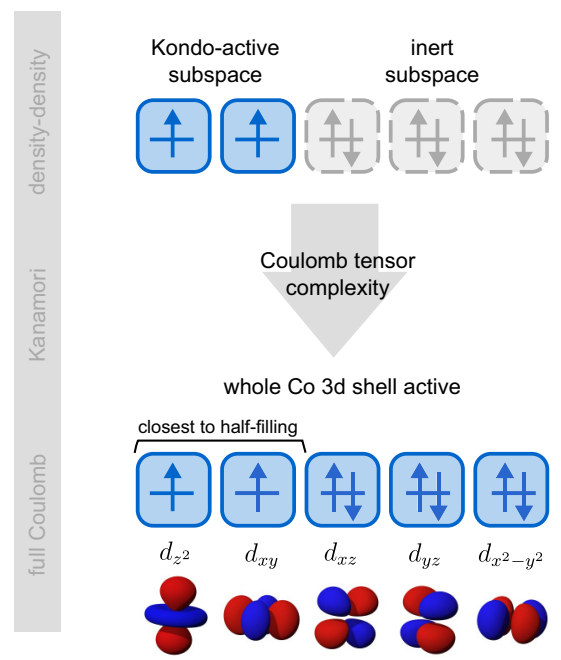

(b)

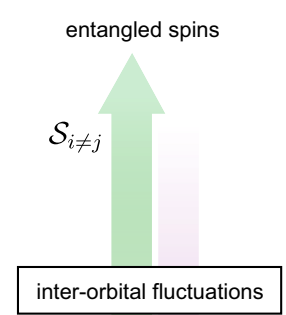

r-orbital fluctuations

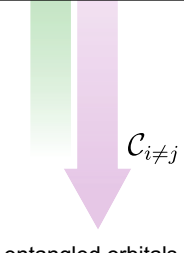

entangled orbitals (c)

Kondo scale $[\mathrm{K}]$

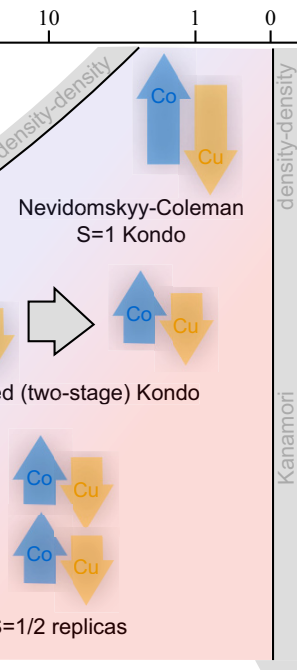

FIG. 3. Schematics of possible Kondo scenarios for Co impurities on Cu hosts. In all three panels (a)-(c), the complexity of the Coulomb tensor increases from top to bottom. (a) Configuration of the Co $3 d$ shell hybridized to the $\mathrm{Cu}(001)$ surface in the presence of Coulomb repulsion. For simplified interactions, the $\left(d_{x y}, d_{z^{2}}\right)$ half-filled orbitals identify the Kondo-active subspace, but for realistic Coulomb tensors the whole multiplet becomes relevant to the Kondo screening. (b) Behavior of the spin $\left(\mathcal{S}_{i \neq j}\right)$ and charge $\left(\mathcal{C}_{i \neq j}\right)$ interorbital fluctuations in relation to the complexity of the Coulomb tensor. Within the density-density approximation, the physics is dominated by the Hund exchange, while exchange interactions (e.g., of the form $U_{i j j k}$ or $U_{i j k l}$ ) favor charge fluctuations and weaken the tendency towards a high-spin state. (c) Kondo screening processes (top to bottom). If the Co impurity is locked in a high-spin state due to the Hund's coupling $J_{H}=U_{i j j i}$ the Nevidomskyy-Coleman scenario [19] can be realized at a very low $T_{K}$. As charge fluctuations are enhanced, an underscreened (or possibly two-stage) Kondo effect may take place at a higher $T_{K}$. The extreme limit for two-orbital models is a pair of $S=1 / 2 \mathrm{Kondo}$ replicas, while in a five-orbital model, the charge redistribution within the whole Co $3 d$ shell can result in a more complicated Kondo effect and in an enhancement of the Kondo scale.

the screening for each parametrization of the Coulomb tensor remains elusive. This is mostly due to the difficulty of observing typical Fermi liquid temperature scaling within our methodology.

\section{RESULTS}

\section{A. Spin correlations and effective local moment}

In order to investigate the screening of the impurity magnetic moment we sample the spin-spin response function in imaginary time within CT-QMC:

$$
\chi_{i j}(\tau)=g^{2}\left\langle\hat{S}_{i}^{z}(\tau) \hat{S}_{j}^{z}(0)\right\rangle,
$$

where $i$ and $j$ denote the Co $3 d$ impurity orbitals, $\hat{S}_{i}^{z}$ is the local spin operator on orbital $i$, and $g$ is the electron spin gyromagnetic factor. The static (i.e., $\omega=0$ ) spin susceptibility is obtained via integration of the diagonal elements of Eq. (7) as

$$
\chi_{i i}(T)=\int_{0}^{\beta} d \tau \chi_{i i}(\tau),
$$

where $\beta$ is the inverse temperature. For a Kondo impurity, the static spin susceptibility follows a Curie-Weiss behavior $\chi(T) \propto 1 / T$ in the local moment regime well above $T_{K}$. As the moment is screened by the conduction electrons, the spin susceptibility has a crossover to a Pauli behavior due to the onset of a Fermi liquid (FL) regime: $\chi^{-1}(T) \propto T+T_{\mathrm{FL}}$, with the characteristic coherence temperature $T_{\mathrm{FL}}$ corresponding to $T_{K}$ in the case of a single impurity $[40,41]$.
In Fig. 4 we compare the spin susceptibility of the $d_{x y}$ and $d_{z^{2}}$ orbitals obtained for all interaction parametrizations. In the corresponding inset we also plot $T \chi_{i i}(T)$, as it is customarily done in order to represent a Curie-Weiss susceptibility as a constant and a Pauli susceptibility as linearly vanishing.
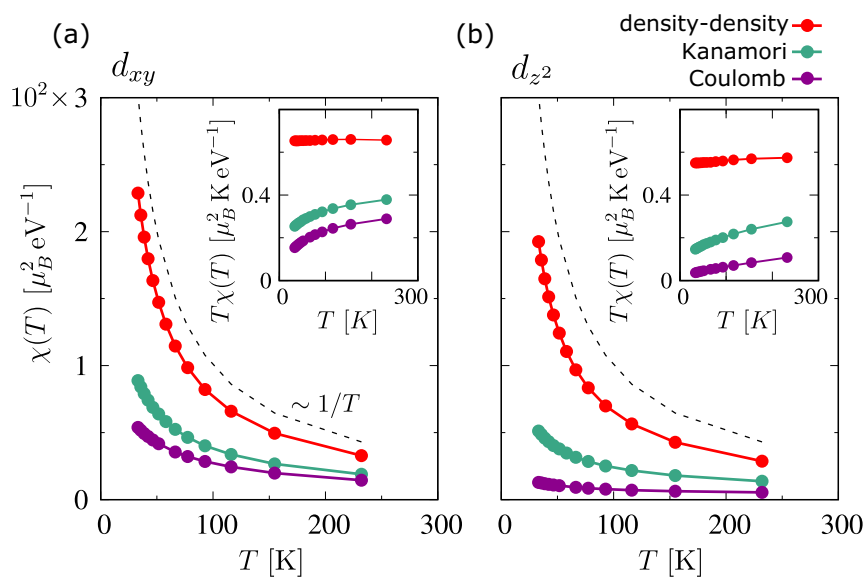

FIG. 4. Orbital-resolved static spin-spin response function $\chi(T) \equiv \chi_{i i}(T)$ for the $d_{x y}$ (a) and the $d_{z^{2}}$ (b) orbitals. The dashed lines show the Curie-Weiss behavior $\chi(T) \propto 1 / T$ in the local moment regime. Plotting $T \chi(T)$ (insets) highlights the differences observed with the three interaction schemes, with $T \chi(T) \sim$ const in the local moment regime, and linearly vanishing at $T \ll T_{K}$ (see text). 
This allows one to highlight the differences observed with the three interaction schemes. Within the density-density approximation, we obtain an almost perfect $1 / T$ behavior of the susceptibilty (in the main panels, and a plateau in the insets) for both orbitals, indicating a Curie-Weiss behavior in the full range of temperatures of our calculations. Consequently, we can infer that the upper bound for the Kondo temperature within the density-density approximation is substantially lower than $30 \mathrm{~K}$, i.e., it is likely of $O(1) \mathrm{K}$. Instead, the Kanamori and full Coulomb parametrizations yield clear signatures of Kondo screening in the same temperature window. The Kanamori coherence scale seems to be still quite low, and at about $30 \mathrm{~K}$ the crossover from a residual entropy to a fully screened moment is indeed far from being complete. We also observe a pronounced departure from a constant $T \chi(T)$ within the Kanamori and-even more evidently-the full Coulomb parametrizations. A linearly vanishing $T \chi(T)$ is clearly observed for the $d_{z^{2}}$ orbital (yet not for the $d_{x y}$ one), which suggests different Kondo scales $T_{K}^{z^{2}}>T_{K}^{x y}$ for those two orbitals. In order to quantify this analysis, we extract $T_{K}$ from the saturation that characterizes the crossover from Curie-Weiss to Pauli behavior of the spin susceptibility. We obtain $T_{K}$ individually for each with the following fitting function $[32,42]$ :

$$
\chi(T)=\frac{\mu^{2}}{3 k_{B}\left(T+2 T_{K}\right)},
$$

where $\mu$ is a fitting parameter of the same order of magnitude of Bohr's magneton $\mu_{B}$. Within the density-density approximation, we estimate $T_{K}<1 \mathrm{~K}$ for both orbitals. A significant enhancement of the Kondo scale is found within the Kanamori, $T_{K}^{x y}=8.5 \mathrm{~K}$ and $T_{K}^{z^{2}}=14 \mathrm{~K}$, and within the full Coulomb, $T_{K}^{x y}=18 \mathrm{~K}$ and $T_{K}^{z^{2}}=40 \mathrm{~K}$, parametrizations. In particular, the latter value is also in qualitative agreement with the estimate of $T_{K} \approx 88 \mathrm{~K}$ from the experiments [5].

Further insight in the different screening processes activated by the Coulomb interaction can be obtained by inspecting two special values of the impurity spin susceptibility in imaginary time: $\chi(\tau=0)$ and $\chi(\tau=\beta / 2)$. At $\tau=0$, it corresponds to the (square of the) bare magnetic moment, sometimes also called the unscreened paramagnetic moment. It indicates the tendency of the Co impurity to build up a quantum magnetic moment at short time scales. Instead, its value at $\tau=\beta / 2$ can be associated to a magnetic moment at asymptotically long times, and hence it provides information on the effectiveness of the dynamical screening due to quantum fluctuations [43]. These two quantities are helpful to visualize the different screening properties within the three interaction schemes and allow us to understand which twobody processes are decisive for the Kondo screening.

In a correlated system we expect a strong contribution from the orbital off-diagonal components of the spin susceptibility and, in particular, in the case under study they are equally important as the diagonal ones. We inspect the screening properties by looking at the total (unscreened and screened, respectively) "effective" spin moment $S_{\text {eff }}$. This involves all components of $\chi_{i j}(\tau)$ and takes into account the difference between the quantum nature of the spin degrees of freedom of the three parametrizations of the Coulomb interaction. Within
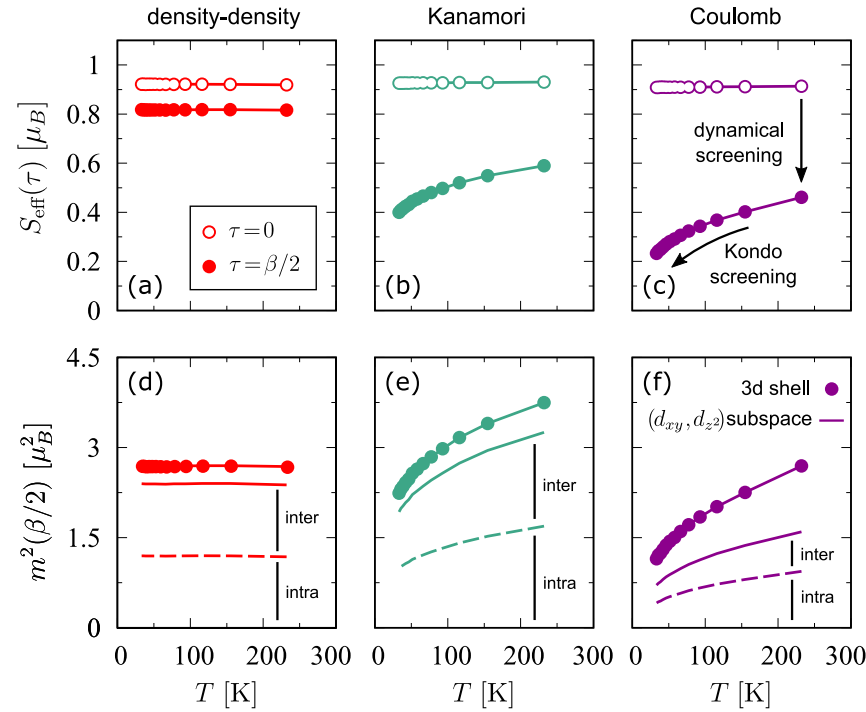

FIG. 5. Analysis of the spin correlations at the Co impurity. (a)-(c) Unscreened (open symbols) and screened (filled symbols) effective spin $S_{\text {eff }}(\tau)$ estimated from the spin susceptibility (see text for the details). (d)-(f) Screened magnetic moment $m^{2}(\beta / 2)$ within the whole $3 d$ shell (filled symbols) and restricted to the $\left(d_{x y}, d_{z^{2}}\right)$ subspace (solid line). The dashed lines separate intra- and interorbital contributions within the subspace. Due to the increasing contribution of orbitals outside the subspace, a two-Kondo-active orbital description of the magnetic moment becomes unsatisfactory for realistic Coulomb interactions.

the density-density approximation we describe an Ising spin, so that the (instantaneous, i.e., $\tau=0$ ) magnetic moment is given by

$$
m_{\text {Ising }}^{2}=g^{2}\left\langle\hat{S}_{z}^{2}\right\rangle
$$

Instead, since the Kanamori and the full Coulomb parametrizations preserve the spin $\mathrm{SU}(2)$ rotational invariance of the Coulomb tensor, the magnetic moment is given by

$$
m_{\text {Heisenberg }}^{2}=g^{2}\left[\left\langle\hat{S}_{x}^{2}\right\rangle+\left\langle\hat{S}_{y}^{2}\right\rangle+\left\langle\hat{S}_{z}^{2}\right\rangle\right]=3 g^{2}\left\langle\hat{S}_{z}^{2}\right\rangle .
$$

We can hence define $m^{2}=\xi \sum_{i j} \chi_{i j}(\tau=0)$, where $\xi=3$, except for the density-density case in which $\xi=1$, and the indices $i$ and $j$ in the summation run over either all Co $3 d$ orbitals or over a subset thereof, as necessary. The natural generalization at finite imaginary time is therefore

$$
m^{2}(\tau)=\xi \sum_{i j} \chi_{i j}(\tau), \quad \text { with } \begin{cases}\xi=1, & \text { Ising, } \\ \xi=3, & \text { Heisenberg, }\end{cases}
$$

which allows us to extract the effective $\operatorname{spin} S_{\text {eff }}(\tau)$ from the relation $m^{2}=g^{2} S_{\text {eff }}^{2}$ for density-density (Ising spin) or $m^{2}=$ $g^{2} S_{\text {eff }}\left(S_{\text {eff }}+1\right)$ for Kanamori and full Coulomb interactions (Heisenberg spin) [43].

The empty symbols in the three upper panels of Fig. 5 show the unscreened (i.e., instantaneous) effective spin $S_{\text {eff }}(\tau=$ 0 ), including the intra- and interorbital contributions from the whole Co $3 d$ shell. For all interaction parametrizations we get an instantaneous paramagnetic spin moment $S_{\text {eff }}>$ 0.9 , in excellent agreement with the value $S=1$ expected in the high-spin configuration, and with the literature [14], 
which remains perfectly constant in the whole range of temperatures considered here. The screened effective moment $S_{\text {eff }}(\tau=\beta / 2)$ at each temperature is suppressed with respect to its $\tau=0$ counterpart by quantum fluctuations. Within the density-density approximation, we observe a sizable effective moment $S_{\text {eff }}(\beta / 2) \approx 0.8$ down to the lowest temperature investigated. This mirrors the information obtained by the analysis of the static spin susceptibility, and substantially rules out any temperature-dependent (i.e., Kondo) screening of the local moment in this temperature window within the density-density approximation. In contrast, within both the Kanamori and the full Coulomb parametrizations we observe the pronounced screening of the "long-time" local moment, which is considerably stronger than what was observed within the density-density approximation. At the same time, a clear temperature dependence of $S_{\text {eff }}$ indicates a strong ability of the environment to Kondo screen the Co impurity spin. Therefore, even at integer filling of the Co $3 d$ shell $\left(n_{d}=8\right)$, the local quantum fluctuations described by more complete parametrizations of the Coulomb interaction destabilize the high-spin state already in the high-temperature regime, and favor the onset of Kondo screening. Instead, this does not happen in the density-density case, for which the Nevidomskyy-Coleman scenario of a strong suppression of $T_{K}$ for a spin $S=1$ is fully realized.

We can analyze the orbital character of the impurity magnetic moment by looking at the (screened) partial magnetic moment (which is an additive quantity, unlike $S_{\text {eff }}$ ). This is obtained by restricting the double sum over $i$ and $j$ in the definition of $m^{2}$ to the $\left(d_{x y}, d_{z^{2}}\right)$ subset of orbitals. We can also distinguish between the $m_{\mathrm{intra}}^{2}(i=j)$ and $m_{\mathrm{inter}}^{2}(i \neq j)$ components within the subspace. As shown in the lower panels of Fig. 5 , within the density-density approximation, $S_{\text {eff }}(\beta / 2)$ is mostly determined by the $\left(d_{x y}, d_{z^{2}}\right)$ subspace, whereas in the full Coulomb parametrizations there is substantial contribution from the $d_{x z}, d_{y z}$, and $d_{x^{2}-y^{2}}$ orbitals. This demonstrates that a two-Kondo-active orbital description of the system is no longer accurate when a realistic Coulomb interaction is taken into account. The intra- and interorbital contributions to the local moment within the $\left(d_{x y}, d_{z^{2}}\right)$ subspace are similar to each other for all three parametrizations, but both are strongly suppressed by introducing interaction terms beyond the density-density approximation. As we discuss in Sec. IV B, this observation can be understood by considering the charge redistribution within the whole Co $3 d$ multiplet, which competes with the spin-locking tendency induced by Hund's coupling.

\section{B. Spin and charge fluctuations}

The full Coulomb tensor (even if here it still assumes a spherical environment) represents the reference point in our comparative analysis, as it gives the most coherent of all the results and the largest Kondo temperature, meaning the closest to the experiments. In order to ascertain the origin of the physical differences between the full Coulomb and the two other approximate schemes we consider the generalized double occupations $\left\langle\hat{n}_{i \sigma} \hat{n}_{j \sigma^{\prime}}\right\rangle$ for parallel $\left(\sigma^{\prime}=\sigma\right)$ and antiparallel $\left(\sigma^{\prime}=\bar{\sigma}\right)$ spin orientations. The numerical data representative of the low-temperature regime (at $T \approx 33 \mathrm{~K}$ ) are collected
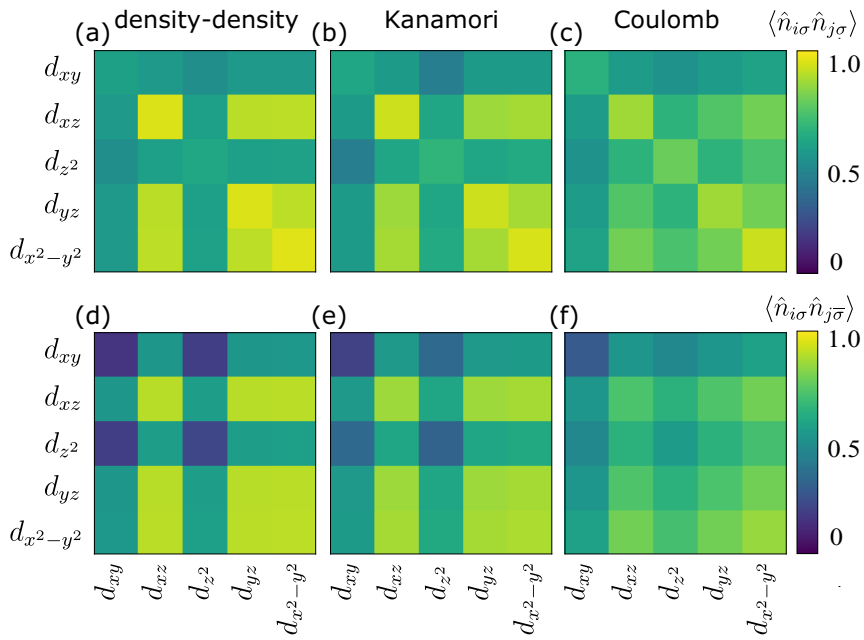

FIG. 6. Generalized double occupations $\left\langle n_{i \sigma} n_{j \sigma^{\prime}}\right\rangle$ for parallel $\left(\sigma^{\prime}=\sigma\right)$ and antiparallel $\left(\sigma^{\prime}=\bar{\sigma}\right)$ spin orientation for the Co $3 d$ multiplet at $T \approx 33 \mathrm{~K}$.

in Fig. 6 and illustrated by a set of matrix heat maps, but their temperature dependence is much weaker than their dependence on the parametrization of the Coulomb interaction. For $\sigma=\sigma^{\prime}$, the diagonal elements correspond to the spinand orbital-resolved occupations $\left\langle\hat{n}_{i \sigma}\right\rangle$. Note that all quantities are symmetrized over both spin $\left(\sigma \leftrightarrow \sigma^{\prime}\right)$ and orbital $(i \leftrightarrow j)$ indices. Within the density-density approximation, both the $d_{x y}$ and $d_{z^{2}}$ orbitals are close to half filling (i.e., $\left\langle\hat{n}_{i \sigma}\right\rangle=0.5$ electrons) and have well defined local moments. All the other Co $3 d$ orbitals are almost full. Moreover, within the $\left(d_{x y}, d_{z^{2}}\right)$ subspace, $\left\langle\hat{n}_{i \sigma} \hat{n}_{j \sigma}\right\rangle \gg\left\langle\hat{n}_{i \sigma} \hat{n}_{j \bar{\sigma}}\right\rangle$, for $i \neq j$, which marks the clear tendency towards an $S=1$ high-spin configuration favored by Hund's coupling $J_{H}=U_{i j j i}$. Within this picture, which is very similar to the atomic ground state configuration $[13,16]$, not only can one identify $d_{x y}$ and $d_{z^{2}}$ as the Kondoactive orbitals, but one could naively expect the physics to be described to a good degree of approximation by a two-orbital AIM, as also assumed in previous literature [16].

The situation is substantially overthrown in the case of the Kanamori and full Coulomb parametrizations. In fact, by progressively including more interaction terms beyond the density-density approximation, i.e., moving from left to right in Fig. 6, two trends emerge clearly. (i) There is a significant charge redistribution within the Co $3 d$ shell. In particular, $\hat{n}_{i \sigma}$ in the $\left(d_{x y}, d_{z^{2}}\right)$ subspace increases as $(0.57,0.60) \rightarrow$ $(0.59,0.65) \rightarrow(0.64,0.77)$, resulting in the suppression of the local moment of the Kondo-active subspace observed in Fig. 5. (ii) The interorbital $(i \neq j)$ double occupations for parallel and antiparallel spin orientations become progressively more similar, i.e., $\left\langle\hat{n}_{i \sigma} \hat{n}_{j \sigma}\right\rangle \simeq\left\langle\hat{n}_{i \sigma} \hat{n}_{j \bar{\sigma}}\right\rangle$ for all pairs of orbitals. As a consequence, the tendency towards a high-spin state of the $\left(d_{x y}, d_{z^{2}}\right)$ pair is substantially weakened. At the same time, the $d_{x y}$ and $d_{z^{2}}$ orbitals still possess the two largest local moments of the entire multiplet, so that they supposedly maintain a prominent role in the Kondo screening process, but with important contributions to the physics coming from the other orbitals. The results are in complete agreement with the conclusions of the spin susceptibility analysis. 

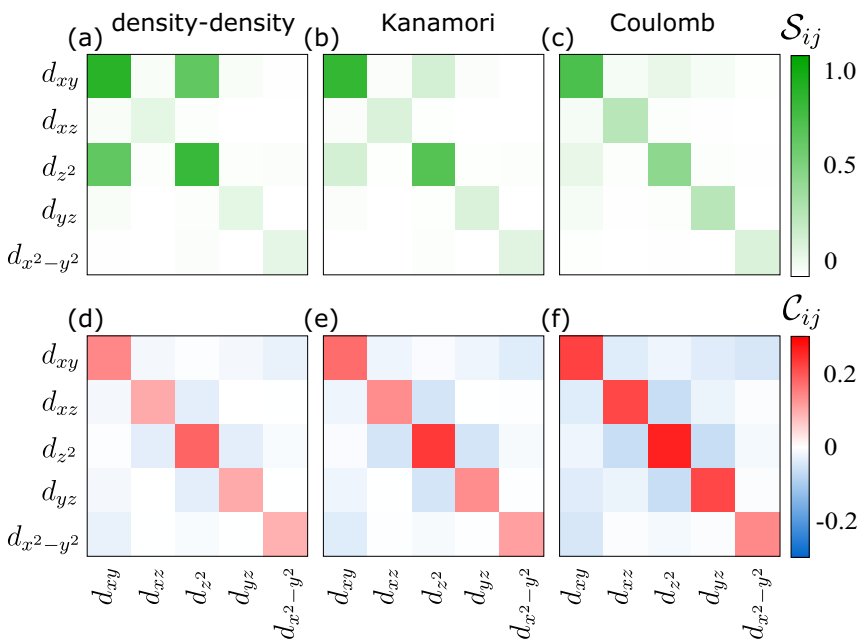

FIG. 7. Correlation functions describing orbital charge $\left(\mathcal{C}_{i j}\right)$ and $\operatorname{spin}\left(\mathcal{S}_{i j}\right)$ fluctuations within the Co $3 d$ shell, calculated at $T \approx 33 \mathrm{~K}$.

The considerations above can also be better understood by explicitly calculating the spin and charge fluctuations, defined as

$$
\begin{aligned}
& \mathcal{S}_{i j}=\left\langle\hat{\sigma}_{i} \hat{\sigma}_{j}\right\rangle-\left\langle\hat{\sigma}_{i}\right\rangle\left\langle\hat{\sigma}_{j}\right\rangle, \\
& \mathcal{C}_{i j}=\left\langle\hat{n}_{i} \hat{n}_{j}\right\rangle-\left\langle\hat{n}_{i}\right\rangle\left\langle\hat{n}_{j}\right\rangle,
\end{aligned}
$$

where we introduced the operators $\hat{n}_{i}=\hat{n}_{i \uparrow}+\hat{n}_{i \downarrow}$ and $\hat{\sigma}_{i}=$ $\hat{n}_{i \uparrow}-\hat{n}_{i \downarrow}$ (and $\left\langle\hat{\sigma}_{i}\right\rangle=0$ in the paramagnetic state). In Fig. 7 we show a matrix heat map for each of the correlators above, for data representative of the low-temperature regime (at $T \approx$ $33 \mathrm{~K}$ ). As usual, we discuss the behavior of spin and charge fluctuations upon increasing the complexity of the Coulomb tensor. The data support the scenario of the destabilization of the high-spin state in the $\left(d_{x y}, d_{z^{2}}\right)$ subspace as both their spin moments (proportional to the elements $S_{i i}$ ) and their interorbital correlator $S_{i \neq j}$ are suppressed, while the spin moments of the orbitals in the rest of the $3 d$ shell increase, as a consequence of the charge redistribution. At the same time, we observe a significant enhancement of charge fluctuations, in both the inter- and the intraorbital components (in absolute value, as $\mathcal{C}_{i \neq j}<0$ ). The orbital spin polarization is responsible for the orbital decoupling in the regime dominated by the Hund exchange [44,45], while the enhancement of the charge fluctuations is the hallmark of increased metallicity in the (Kanamori and) full Coulomb parametrization(s), as also previously reported in model studies of multiorbital impurity problems [46]. The high-spin state is weakened already by the spin-flip term in the Kanamori Hamiltonian, but the two-body mixing terms, involving combinations of three (e.g., $\left.U_{i j j k}\right)$ or even four $\left(U_{i j k l}\right)$ different orbital indices, which are included within the full Coulomb parametrization, are highly effective in reducing the "orbital rigidity" and eventually yield a solution which is well described neither by a single $S=1$ Kondo effect [19] nor by two independently screened $S=1 / 2$ spins $[14,16]$. A thorough discussion of these terms and their relation with Hund's coupling is provided in Appendix B.

Interestingly, the temperature dependence of both spin and charge fluctuations within the Co $3 d$ shell is negligible with
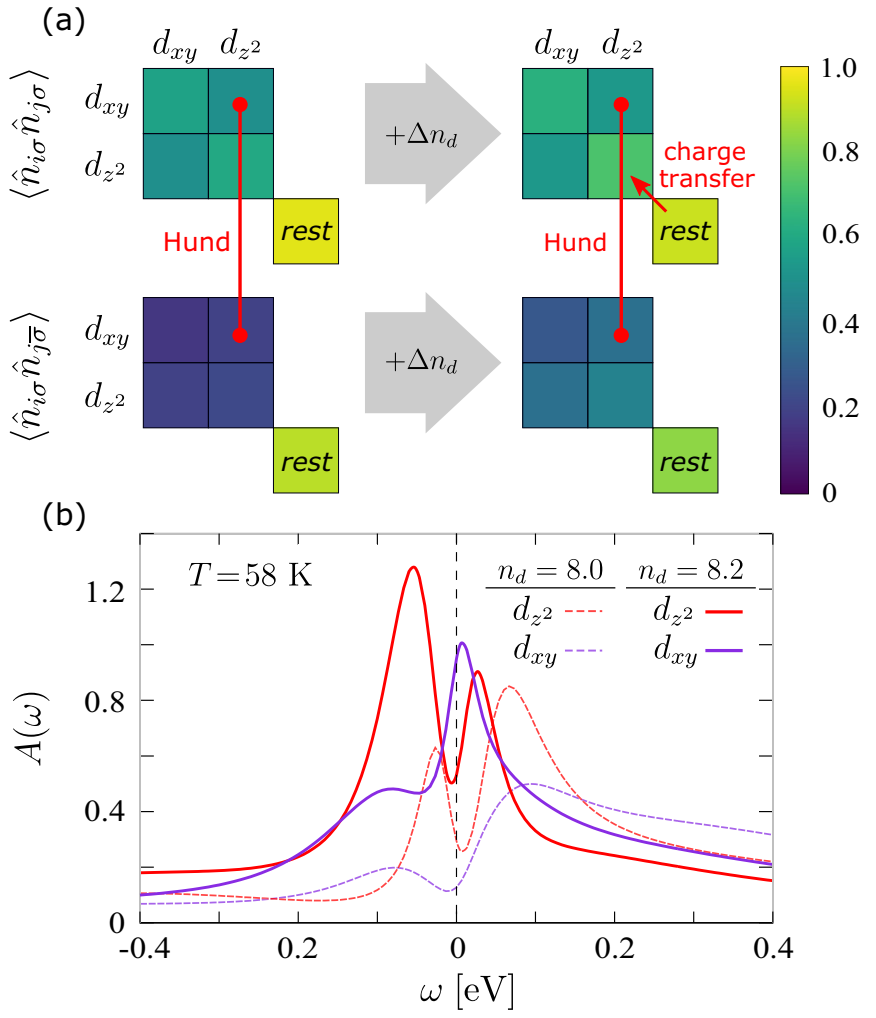

FIG. 8. (a) Generalized double occupations within the densitydensity approximation. The $2 \times 2$ block represents the $\left(d_{x y}, d_{z^{2}}\right)$ subspace, while the extra block, labeled "rest," denotes the average over the diagonal elements for the $\left(d_{y z}, d_{x z}, d_{x^{2}-y^{2}}\right)$ subspace. Moving away from integer filling $n_{d}=8$ results in a net charge transfer (in addition to the extra $\Delta n_{d}=0.2$ electrons) to the $\left(d_{x y}, d_{z^{2}}\right)$ subspace from the rest of the multiplet. The overall effect is a weakening of the tendency towards the $S=1$ high-spin state. (b) Spectral function $A(\omega)$ of the $d_{x y}$ and $d_{z^{2}}$ orbitals within the density-density approximation. The development of low-energy resonances away from integer filling is compatible with an enhancement of the Kondo scale.

respect to the changes observed between different interaction schemes, so that the above picture is valid in the whole range $300-30 \mathrm{~K}$, and probably still holds below that.

\section{Spectral signatures of the Kondo effect}

Useful insight can also be obtained by looking at the orbital-resolved spectral function of the Co $3 d$ shell. While one may estimate $T_{K}$ (or at least an apparent $T_{K}$ at $T \neq 0$ ) from spectral features such as the width of the resonance $[14,16]$, we will refrain from doing so. Since our spectral functions are obtained with a numerical analytic continuation procedure (maximum entropy method), we only take them as qualitative indications of the redistribution of the spectral weight.

First, we consider results obtained within the densitydensity approximation, which are shown in Fig. 8. For a Co $3 d$ shell occupation of $n_{d}=8.0$ and at $T=58 \mathrm{~K}$, neither the $d_{x y}$ nor the $d_{z^{2}}$ orbital displays a resonant feature close to the Fermi level, in agreement with the lack of Kondo screening. By adjusting to $n_{d}=8.2$, corresponding to a charge transfer from the $\mathrm{Cu}$ surface to the $\mathrm{Co}$ adatom, prominent resonances 


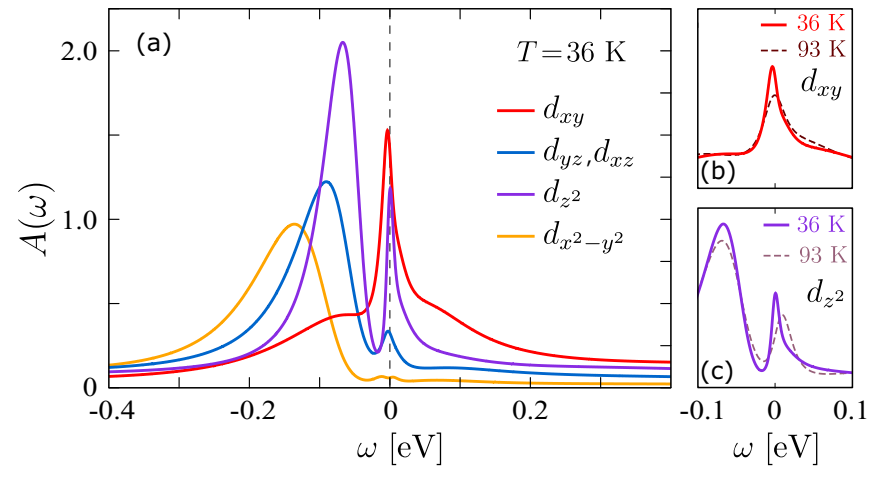

FIG. 9. (Main panel) Orbital-resolved spectral function of the Co $3 d$ shell at integer filling, i.e., $n_{d}=8$ electrons in the full Coulomb parametrization. The $d_{y z}$ and $d_{x z}$ resonances suggest that those orbitals may be relevant to the Kondo effect. (Side panels) Temperature evolution of the $d_{x y}$ and $d_{z^{2}}$ low-energy resonances.

appear in the spectral functions of both orbitals. The analysis of the charge redistribution within the $3 d$ shell (upper panels of Fig. 8) shows that, upon adding the extra $\Delta n_{d}=0.2$ electrons, the occupation of the Kondo-active orbitals increases as $(0.57,0.60) \rightarrow(0.63,0.71)$. However, part of the charge accumulating in the subspace comes from the rest of the Co $3 d$ shell. This is indicated by the red arrow in Fig. 8, where the extra block denotes the average occupation of the $\left(d_{y z}, d_{x z}, d_{x^{2}-y 2}\right)$ subspace. Such a charge redistribution is detrimental to the stabilization of the high-spin state, which one realizes by comparing the interorbital double occupations $\left\langle\hat{n}_{i \sigma} \hat{n}_{j \sigma}\right\rangle$ and $\left\langle\hat{n}_{i \sigma} \hat{n}_{j \bar{\sigma}}\right\rangle$ (connected by red dots and a line in the upper panels of Fig. 8). The effect of charging is qualitatively analogous to, yet not as strong as, what we observed by comparing the density-density and full Coulomb parametrizations at $n_{d}=8.0$ in Fig. 6. Hence both scenarios are compatible with an enhancement of the Kondo scale.

A clear signature of the Kondo effect is indeed observed already at integer filling in the full Coulomb spectral function, which is shown in Fig. 9 for different temperatures. A clear resonance close to the Fermi level is observed for both the $d_{x y}$ and the $d_{z^{2}}$ orbitals. The resonance is already present at $T \approx 100 \mathrm{~K}$, but it gets progressively closer to the Fermi level and its width decreases as the temperature is lowered (see side panels of Fig. 9). Interestingly, within this interaction scheme, a low-energy resonance develops also in the $\left(d_{x z}, d_{y z}\right)$ doublet. This feature is almost completely absent within the density-density approximation, and it can be regarded as a further indication that a purely $\left(d_{x y}, d_{z^{2}}\right)$ description of the Kondo effect is not adequate, when accounting for a realistic Coulomb interaction in $\mathrm{Co} / \mathrm{Cu}(001)$. Similar resonances are also evident in the spectral functions obtained away from integer filling (not shown), where the role of the other three orbitals is possibly enhanced.

\section{DISCUSSION AND CONCLUSIONS}

In this work we investigate the Kondo screening properties of $\mathrm{Co} / \mathrm{Cu}(001)$ in its full realistic complexity. We solve an AIM for the whole Co $3 d$ shell and we focus on the role of the parametrization of the Coulomb tensor for the Kondo effect.
It is important to compare our findings to previous studies in the literature, in order to highlight both the differences and the similarities.

Previous theoretical analyses were restricted to a twoorbital model for the Kondo-active orbitals [16], with approximate interaction schemes $[14,16]$ or impurity solvers [14]. The most direct comparison can be done with the results reported by Jacob [14], obtained with similar interaction parameters as ours, derived from first principles within the constrained random-phase approximation. There, many-body effects are taken into account at the level of the one-crossing approximation (OCA), in contrast to our numerically exact CT-QMC. The OCA calculation takes into account all density-density terms as well as the spin-flip contributions. It may therefore be regarded as an intermediate parametrization between density-density and Kanamori, albeit restricted to one-crossing diagrams. There, a Kondo feature for the $d_{z^{2}}$ orbital at $T \sim 10 \mathrm{~K}$ for $\mathrm{Co} / \mathrm{Cu}(001)$ is reported, with a Kondo temperature $T_{K} \approx 90 \mathrm{~K}$, estimated from the width of the Kondo resonance in the spectral function. The lack of a similar feature for the other Kondo-active orbital $\left(d_{x y}\right.$ in the notation of this work) was suggested as evidence of an underscreened Kondo effect. Whether the Co magnetic moment is completely screened at lower temperature, with the onset of a Fermi liquid state and the realization of a two-stage Kondo effect, was not investigated, and it remains debatable.

On the basis of our CT-QMC results we can delineate a quite different situation, whose physical explanation can be unveiled thanks to our comparative analysis of the various Coulomb tensor parametrizations. Within the density-density approximation the overall $T_{K}$ is much smaller than the lowest temperature of our calculation, and the NevidomskyyColeman scenario for a spin $S=1$ Kondo is fully realized. We progressively include additional exchange interactions within the Co $3 d$ multiplet in the Kanamori and eventually all of them in the full Coulomb parametrizations. Due to the associated charge redistribution, spin fluctuations are partially quenched, whereas charge fluctuations increase, together with the orbital entanglement. Two effects consequently emerge. The $d_{x y}$ and $d_{z^{2}}$ orbitals start to thrive on Kondo screening, especially with the full Coulomb interaction, while the remaining three $d$ orbitals substantially increase their active contribution to the local moment. The latter is transparently observed by comparing the charge distribution within the Co $3 d$ multiplet in Fig. 6 and the contribution of the $\left(d_{x y}, d_{z^{2}}\right)$ subspace to the local moment in Fig. 5 (by moving from the left to the right panels). The relevant role of the whole Co $3 d$ shell within the full Coulomb parametrization of the interaction has also been suggested in the past $[15,23]$. However, the temperature regime previously investigated is hardly relevant for extracting useful information about the Kondo screening.

The outcome of the present study therefore changes the conventional interpretation of the Kondo effect in the prototypical Co-adatom systems, once a realistic interaction tensor is properly taken into account. Since the whole $3 d$ shell is involved in the Kondo screening, one neither has a Nevidomskyy-Coleman scenario with the screening of a $S=$ 1 spin at low temperatures nor two independent $S=1 / 2$ spin Kondo replicas in the $\left(d_{x y}, d_{z^{2}}\right)$ subspace. The most appropriate way of describing the Kondo effect in Co adatoms on a 
$\mathrm{Cu}(001)$ surface is, as a matter of fact, a multiorbital entangled correlated state. While two of the five $3 d$ orbitals have the largest magnetic moment and a favorable hybridization to the substrate in order to display clear Kondo peaks, they are not decoupled enough from the other orbitals to allow for an effective two-orbital description of the Co $3 d$ shell.

Finally, we note that some details of the calculations may differ from other results in the literature. For instance, Jacob [14] and Baruselli et al. [16] consider STM geometries, where the STM tip also consists of a $\mathrm{Cu}$ pyramid grown in the (001) direction-or the (111) direction when considering $\mathrm{Co} / \mathrm{Cu}(111)$. In some cases $[8,14,47]$, besides the $\mathrm{Co}-\mathrm{Cu}$ adsorption distance, also the atomic positions of some $\mathrm{Cu}$ atoms of the surface layer are relaxed. Despite these effects possibly being important, we are confident that the differences observed within the different parametrization of the Coulomb interaction influence the Kondo screening in a more fundamental way than the details of the DFT calculations.

To conclude, we revisited the prototypical $\mathrm{Co} / \mathrm{Cu}(001)$ Kondo problem under a new light. We established how the parametrization of the Coulomb tensor affects the screening of the impurity magnetic moment, and we highlight the active role of the whole Co $3 d$ shell in the Kondo effect. Our analysis is likely relevant and can be extended to other Kondo systems with transition metal adatoms.

\section{ACKNOWLEDGMENTS}

We thank A. Amaricci, M. Capone, L. Fanfarillo, L. de' Medici, A. Nevidomskyy, M. Schüler, and T. O. Wehling for insightful discussions. We are also grateful to D. Jacob for critical reading of the manuscript. M.P.B. and C.H. acknowledge the German Research Foundation (DFG) for funding via Project No. HE 5675/6-1, the high-performance-computing team of the Regionales Rechenzentrum at Universität Hamburg, and the North-German Supercomputing Alliance (HLRN) for computational resources. A.K. and G.S. are supported by DFG-SFB 1170 Tocotronics, and further acknowledge financial support from the DFG through the Würzburg-Dresden Cluster of Excellence on Complexity and Topology in Quantum Matter ct.qmat (EXC 2147, Project-id No. 390858490). We gratefully acknowledge the Gauss Centre for Supercomputing e.V. [48] for funding this project by providing computing time on the GCS Supercomputer SuperMUC at Leibniz Supercomputing Centre [49]. This research was supported in part by the National Science Foundation under Grant No. NSF PHY-1748958. A.V. acknowledges financial support from the Austrian Science Fund (FWF) through the Erwin Schrödinger fellowship No. J3890-N36, through Project "LinReTraCe" No. P 30213, and Project No. P 31631.

\section{APPENDIX A: DETAILS AND PHYSICAL IMPLICATIONS OF THE $k$-MESH CONVERGENCE OF THE Cu(001) SURFACE}

Throughout our study, we realized that the physical picture of $\mathrm{Co} / \mathrm{Cu}(001)$ delicately depends on the size of the $k$ mesh of the Brillouin zone. In Fig. 10 we show the orbital-resolved hybridization function $\Delta\left(\iota \omega_{n}\right)$ describing the embedding of
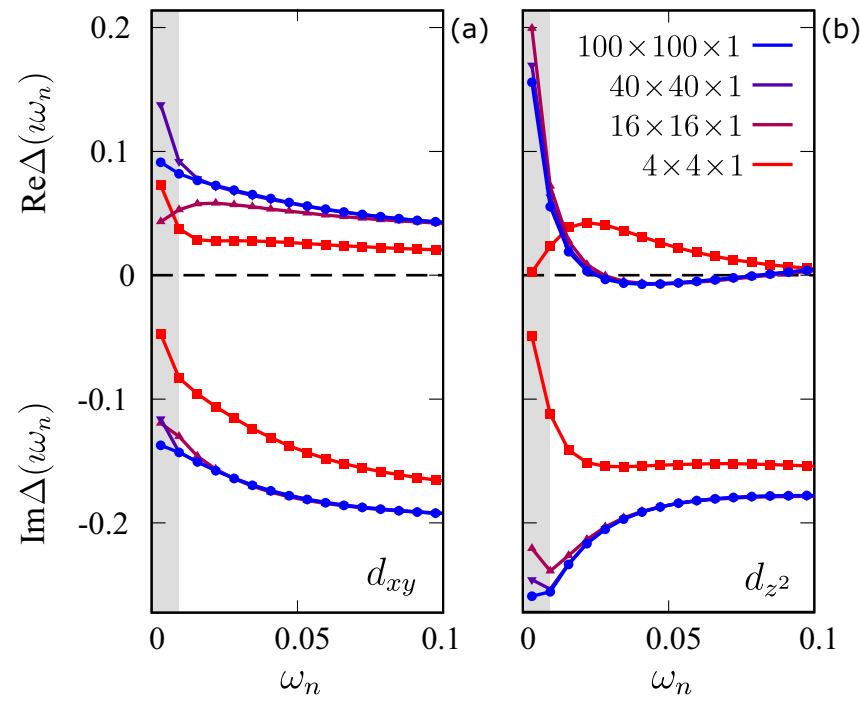

FIG. 10. Real and imaginary parts of the hybridization function of the Co $d_{x y}$ (a) and $d_{z^{2}}$ (b) orbitals in the Matsubara representation: $\omega_{n}=(2 n+1) \pi / \beta$, with an infrared cutoff $\propto \beta=1000 \mathrm{eV}^{-1}$. When evaluated on different $k$-mesh sampling of the Brillouin zone, $\Delta\left(l \omega_{n}\right)$ shows a slow convergence with the mesh size, in particular for the $d_{z^{2}}$ orbital.

the $\mathrm{Co}$ adatom on the $\mathrm{Cu}$ surface. We compare the results obtained for different $k$ meshes (all centered around the $\Gamma$ point). We find that $\Delta\left(\imath \omega_{n}\right)$ displays a slow convergence with the size of the $k$ mesh, in particular for the $d_{z^{2}}$ orbital. For the sparsest mesh considered, i.e., $4 \times 4 \times 1 k$ points, $\Delta\left(\imath \omega_{n}\right)$ at low frequencies displays a qualitatively different behavior for the $d_{z^{2}}$ orbital when compared to more accurate meshes (with up to $100 \times 100 \times 1 k$ points), while for the $d_{x y}$ orbital the differences are mainly quantitative. Since the differences are observed at relatively low energy scales, it is possible that this effect may be overlooked in calculations with a low energy resolution, or with a large smearing parameter $\eta$ in the hybridization function $\Delta(\omega+\imath \eta)$. Differences between the $40 \times 40 \times 1$ and $100 \times 100 \times 1 k$-point meshes can be observed on energy scales $<0.01 \mathrm{eV}$, which corresponds approximatively to the lowest Matsubara frequency for the lowest temperatures of our QMC calulations, $\beta=350 \mathrm{eV}^{-1}$ (shaded area in Fig. 10).

However, here we show that this seemingly technical detail can have drastic consequences on the physical description of the system. For instance, we can consider the temperature evolution of the lowest Matsubara frequency $\omega_{0}$ of the electronic self-energy, which in a Fermi liquid should scale as $\operatorname{Im} \Sigma\left(\iota \omega_{0}\right) \propto T$ (see, e.g., Refs. [41,50]). In Fig. 11 we show $\operatorname{Im} \Sigma\left(\iota \omega_{0}\right)$ for both the $d_{x y}$ and $d_{z^{2}}$ orbitals within the densitydensity approximation. The Fermi liquid scaling seems to be recovered at low-enough temperatures for the sparsest $4 \times 4 \times 1 k$ mesh, for both orbitals. For denser $k$ meshes, and in particular for the $100 \times 100 \times 1$ one, the self-energy of the $d_{z^{2}}$ orbital displays a clear non-Fermi liquid behavior, which we follow down to $T \approx 33 \mathrm{~K}$.

Finally, in Fig. 12 we compare $\operatorname{Im} \Sigma\left(\iota \omega_{0}\right)$ obtained within all parametrizations of the Coulomb tensor. We note that only the full Coulomb case seems to be compatible with a linear 

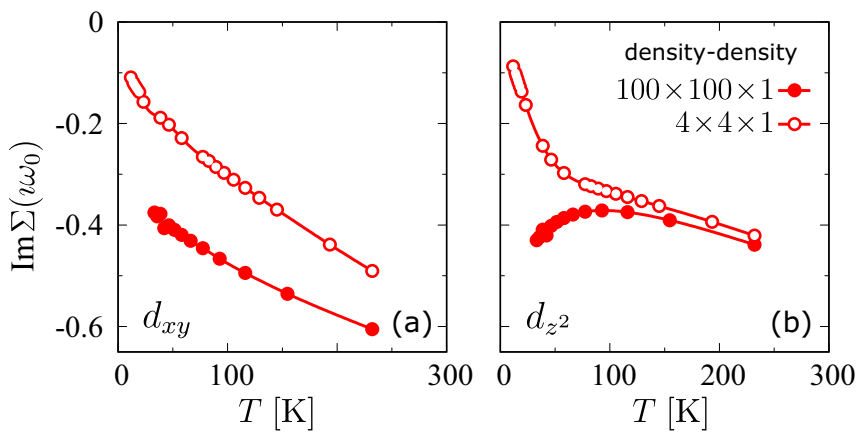

FIG. 11. Temperature evolution of $\operatorname{Im} \Sigma\left(\imath \omega_{0}\right)$ for the Co $d_{x y}$ and $d_{z^{2}}$ orbitals within the density-density approximation. A sparse $4 \times$ $4 \times 1 k$ mesh suggests the onset of a Fermi-liquid regime at relatively high temperature, whereas strongly non-Fermi liquid features emerge for denser meshes.

behavior, although this feature alone is not enough to confirm the onset of a Fermi liquid state at low temperatures. This observation is important because, in the literature, calculations for $\mathrm{Co} / \mathrm{Cu}(001)$ performed without including the full Coulomb tensors $[14,16]$ indicated the Kondo screening to be the most effective for the $d_{z^{2}}$ orbital. Our calculations show that, with an accurate-enough description of the hybridization between the $\mathrm{Co}$ adatom and the $\mathrm{Cu}$ surface, and at low-enough temperatures, the density-density approximation does not confirm this picture. It is instead necessary to take into account more realistic forms of the Coulomb interaction to obtain estimates of the Kondo scale comparable with the experimental observations.

\section{APPENDIX B: COULOMB TENSOR}

The full Coulomb interaction Hamiltonian for the impurity model is given by

$$
\hat{H}_{\mathrm{C}}=\frac{1}{2} \sum_{m m^{\prime} m^{\prime \prime} m^{\prime \prime \prime}} \sum_{\sigma \sigma^{\prime}} U_{i j k l} \hat{d}_{m \sigma}^{\dagger} \hat{d}_{m^{\prime} \sigma^{\prime}}^{\dagger} \hat{d}_{m^{\prime \prime \prime} \sigma^{\prime}} \hat{d}_{m^{\prime \prime} \sigma},
$$

where $U_{m m^{\prime} m^{\prime \prime} m^{\prime \prime \prime}}$ is the Coulomb tensor, labeled by the orbital momentum quantum number $m=-\ell, \ldots, 0, \ldots, \ell$ (in this case, $\ell=2$ for the Co $3 d$ shell). For a spherically symmetric atom, the Coulomb tensor can be expressed as follows
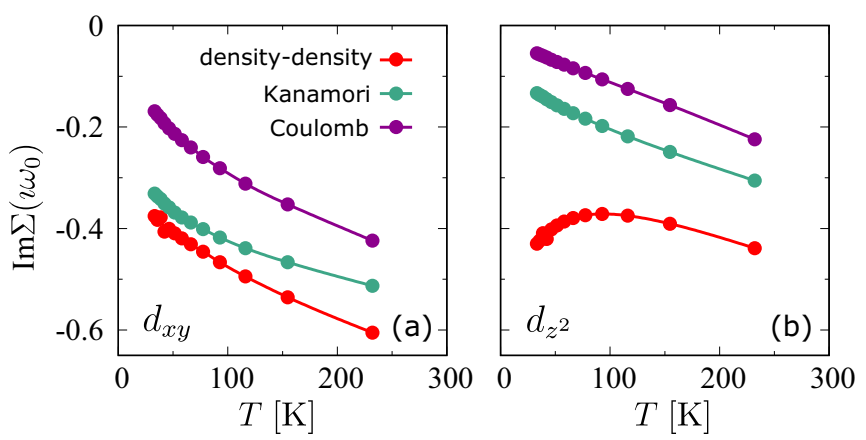

FIG. 12. Temperature evolution of $\operatorname{Im} \Sigma\left(\iota \omega_{0}\right)$ for the Co $d_{x y}$ and $d_{z^{2}}$ orbitals and different parametrization schemes of the Coulomb interaction for the $100 \times 100 \times 1 k$ mesh.
[29,31]:

$$
U_{m m^{\prime} m^{\prime \prime} m^{\prime \prime \prime}}=\sum_{k=0}^{2 \ell} a_{k}\left(m m^{\prime} ; m^{\prime \prime} m^{\prime \prime \prime}\right) F^{k},
$$

where the coefficients $a_{k}$ and the Slater parameters $F^{k}$ are given by integrals of spherical harmonics and the radial part of the wave function, respectively. Their expressions are well known and can be found, e.g., in Refs. [29,31,33]. In the basis of the spherical harmonics, the Coulomb tensor includes all two-, three-, and four-index interaction terms which separately fulfill the conservation of both spin and angular momentum [29]. The first condition is encoded in the choice of the spin indices of Hamiltonian (B1), while the latter reads $m+m^{\prime}=m^{\prime \prime}+m^{\prime \prime \prime}$.

For actual calculations it is convenient to rotate the Coulomb tensor $U_{m m^{\prime} m^{\prime \prime} m^{\prime \prime \prime}} \rightarrow U_{i j k l}$ from spherical to cubic harmonics. The corresponding transformation $(m>0)$ for the basis functions is given by

$$
\begin{aligned}
K_{\ell}^{m} & =\frac{1}{\sqrt{2}}\left[(-1)^{m} Y_{\ell}^{m}+Y_{\ell}^{-m}\right], \\
K_{\ell}^{0} & =Y_{0}^{\ell}, \\
K_{\ell}^{-m} & =\frac{1}{l \sqrt{2}}\left[(-1)^{m} Y_{\ell}^{m}-Y_{\ell}^{-m}\right] .
\end{aligned}
$$

In the case of the Co $3 d$ shell we label the cubic harmonics as $\left(K_{2}^{-2}, K_{2}^{-1}, K_{2}^{0}, K_{2}^{1}, K_{2}^{2}\right)=\left(d_{x y}, d_{x z}, d_{z^{2}}, d_{y z}, d_{x^{2}-y^{2}}\right)$. A data file containing the full Coulomb tensor in this basis, which we used in all of our numerical calculations, is also provided as Supplemental Material [51].

\section{One- and two-index interaction terms}

The terms of the Coulomb tensor $U_{i j k l}$ which contain only two different indices can be classified as follows: the densitydensity terms, which include the intraorbital interaction $U_{i i i i}$ (existing only for $\sigma \neq \sigma^{\prime}$ due to the Pauli exclusion principle) and the interorbital interaction $U_{i j i j}=\left(U_{i i i i}+U_{j j j j}\right) / 2-$ $2 U_{i j j i}$, where $U_{i j j i}$ represents the Hund's exchange coupling for parallel spin configurations [14]. Other additional exchange terms, for opposite spin configurations, account for spin-flip and pair hopping processes, with values $U_{i j j i}$ and $U_{i i j j}$, which are the same as the density-density exchange in the cubic harmonics basis.

It is possible to take all previous terms into account in a relatively compact form, giving rise to the Kanamori interaction Hamiltonian [see also Eqs. (4) and (5)]

$$
\begin{aligned}
\hat{H}_{\mathrm{K}}= & \sum_{i} U_{i i} \hat{n}_{i \uparrow} \hat{n}_{i \downarrow}+\sum_{i \neq j} \sum_{\sigma \sigma^{\prime}}\left(U_{i j}-J_{i j} \delta_{\sigma \sigma^{\prime}}\right) \hat{n}_{i \sigma} \hat{n}_{j \sigma^{\prime}} \\
& +\sum_{i \neq j} J_{i j}\left(\hat{d}_{i \uparrow}^{\dagger} \hat{d}_{j \downarrow}^{\dagger} \hat{d}_{i \downarrow} \hat{d}_{j \uparrow}-\hat{d}_{i \uparrow}^{\dagger} \hat{d}_{i \downarrow}^{\dagger} \hat{d}_{j \uparrow} \hat{d}_{j \downarrow}\right),
\end{aligned}
$$

which is defined in terms of the two-index interactions $U_{i i}=$ $U_{i i i i}, U_{i j}=U_{i j i j}$, and $J_{i j}=U_{i j j i}$. One can reduce to only two parameters, $U$ and $J$, by expressing the interaction in terms of the Slater integrals $F^{0}, F^{2}$, and $F^{4}$, as

$$
U_{0}=F^{0}+\frac{8}{7} \frac{1}{14}\left(F^{2}+F^{4}\right)
$$


TABLE II. Intra- and interorbital interactions of density-density type in the spherically symmetric Coulomb tensor.

\begin{tabular}{lccccc}
\hline \hline$U_{i j i j}$ & $d_{x y}$ & $d_{x z}$ & $d_{z^{2}}$ & $d_{y z}$ & $d_{x^{2}-y^{2}}$ \\
\hline$d_{x y}$ & $U_{0}$ & $U_{0}-2 J_{1}$ & $U_{0}-2 J_{2}$ & $U_{0}-2 J_{1}$ & $U_{0}-2 J_{3}$ \\
$d_{x z}$ & & $U_{0}$ & $U_{0}-2 J_{4}$ & $U_{0}-2 J_{1}$ & $U_{0}-2 J_{1}$ \\
$d_{z^{2}}$ & & & $U_{0}$ & $U_{0}-2 J_{4}$ & $U_{0}-2 J_{2}$ \\
$d_{y z}$ & & & & $U_{0}$ & $U_{0}-2 J_{1}$ \\
$d_{x^{2}-y^{2}}$ & & & & & $U_{0}$ \\
\hline \hline
\end{tabular}

$$
\begin{gathered}
J_{1}=\frac{1}{49}\left(3 F^{2}+\frac{20}{9} F^{4}\right), \\
J_{2}=-2 \frac{5}{7} \frac{1}{14}\left(F^{2}+F^{4}\right)+3 J_{1}, \\
J_{3}=6 \frac{5}{7} \frac{1}{14}\left(F^{2}+F^{4}\right)-5 J_{1}, \\
J_{4}=4 \frac{5}{7} \frac{1}{14}\left(F^{2}+F^{4}\right)-3 J_{1},
\end{gathered}
$$

and identifying $U=F^{0}$ and $J=\frac{1}{14}\left(F^{2}+F^{4}\right)$ with an almost constant ratio $F^{4} / F^{2} \approx 0.625$ for $3 d$ ions [52]. See also, e.g., Refs. [32,33] for a related discussion.

All two-index terms in the basis of the $3 d$ cubic harmonics are summarized in Tables II and III, for reference. In Fig. 13 we show a schematic representation of all possible two-index interaction terms (excluding permutations) for the $3 d$ shell in the basis of the cubic harmonics.

For our spherically symmetric calculations of the $\mathrm{Co} / \mathrm{Cu}(001)$ system, we set $U=4.0 \mathrm{eV}$ and $J=0.9 \mathrm{eV}$, which characterize the interaction matrix with Slater integrals $F^{0}=4.0 \mathrm{eV}, F^{2} \simeq 7.75 \mathrm{eV}$, and $F^{4} \simeq 4.85 \mathrm{eV}$, and result in the interaction parameters $U_{0}=5.02 \mathrm{eV}, J_{1}=0.69 \mathrm{eV}$, $J_{2}=0.80 \mathrm{eV}, J_{3}=0.39 \mathrm{eV}$, and $J_{4}=0.49 \mathrm{eV}$.

In the case of a nonspherical Coulomb tensor, some symmetries between the interaction terms are lifted. For instance, symmetries between all pairs of $d_{x y}, d_{x z}$, and $d_{y z}$ orbitals, or between the $d_{z^{2}}$ and any of the two planar orbitals $\left(d_{x y}\right.$ and $d_{x^{2}-y^{2}}$ ). Moreover, the intraorbital term $U_{\text {iiii }}$ becomes orbital dependent. The nonspherical interaction parameters for $\mathrm{Co} / \mathrm{Cu}(001)$ have been evaluated with the constrained random phase approximation, in e.g., Ref. [14].

TABLE III. Hund's exchange couplings of density-density and Kanamori type in the spherically symmetric Coulomb tensor.

\begin{tabular}{lccccc}
\hline \hline$U_{i j j i}$ & $d_{x y}$ & $d_{x z}$ & $d_{z^{2}}$ & $d_{y z}$ & $d_{x^{2}-y^{2}}$ \\
\hline$d_{x y}$ & $J_{1}$ & $J_{2}$ & $J_{1}$ & $J_{3}$ \\
$d_{x z}$ & & $J_{4}$ & $J_{1}$ & $J_{1}$ \\
$d_{z^{2}}$ & & & $J_{4}$ & $J_{2}$ \\
$d_{y z}$ & & & & $J_{1}$ \\
$d_{x^{2}-y^{2}}$ & & & & & \\
\hline \hline
\end{tabular}

(a)

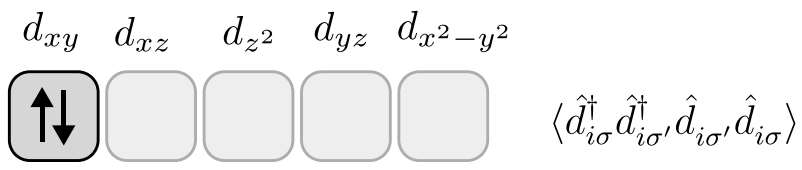

(b)

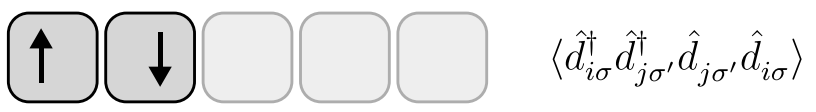

(c)
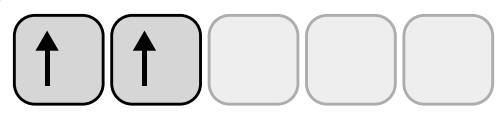

$\left\langle\hat{d}_{i \sigma}^{\dagger} \hat{d}_{j \sigma}^{\dagger} \hat{d}_{j \sigma} \hat{d}_{i \sigma}\right\rangle$

(d)

(e)

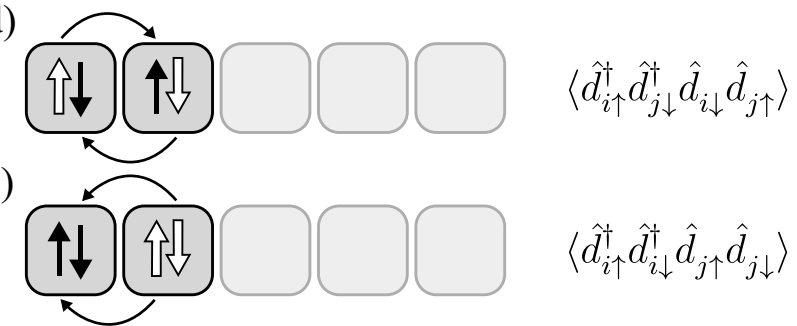

FIG. 13. Schematic representation of the two-index interaction terms (excluding permutations) for the $3 d$ shell in the basis of the cubic harmonics. The white and black spins denote the initial and final configurations connected by the operator, respectively. The terms are (a) intraorbital interaction, (b), (c) interorbital interactions, including the Hund's exchange for parallel spin configurations, (d) spin flip, and (e) pair hopping.

\section{Three- and four-index interaction terms}

Even though the Kanamori parametrization restores the rotational invariance of the Coulomb tensor (in the spherical approximation) and provides an exact parametrization for two- and three-orbital models, it does not contain all possible interaction terms allowed for the whole $3 d$ shell.

In the basis of the spherical harmonics, there exist threeindex terms for the form $U_{m m m^{\prime} m^{\prime \prime}}$ and $U_{m^{\prime} m^{\prime \prime} m m}$ such that $2 m=m^{\prime}+m^{\prime \prime}$. All other terms, e.g., $U_{m m^{\prime} m^{\prime \prime} m}$ or $U_{m^{\prime} m m m^{\prime \prime}}$, can only conserve the angular momentum if $m^{\prime}=m^{\prime \prime}$, giving rise to two-index terms already included in the Kanamori parametrization. These interactions can be interpreted in terms of the creation or annihilation of an orbital pair. However, once rotated in the cubic harmonics basis, besides the pair creation $\left(U_{j j i k}\right)$ or annihilation $\left(U_{i k j j}\right)$ terms, one also obtains terms associated to matrix elements $U_{j i j k}$ and $U_{j i k j}\left(\sigma=\sigma^{\prime}\right)$. Similarly, in the only four-index terms $U_{m m^{\prime} m^{\prime \prime} m^{\prime \prime \prime}}$ that are allowed, the pair of creation operators can only carry angular momentum $m+m^{\prime}= \pm 1$ or $m+m^{\prime}=0(m \neq 0)$, which is mirrored by the annihilation operators $m^{\prime \prime}+m^{\prime \prime \prime}$. These interactions resemble hopping of unpaired electrons involving four different orbitals both in the spherical and in the cubic harmonics basis. In Fig. 14 we show a schematic representation of three- and four-index interaction terms (excluding permutations) for the $3 d$ shell in the basis of the cubic harmonics.

A complete "analytic" parametrization of all possible three- and four-index terms, in analogy to the standard one for the two-index terms, is out of the scope of this work. 
(a)

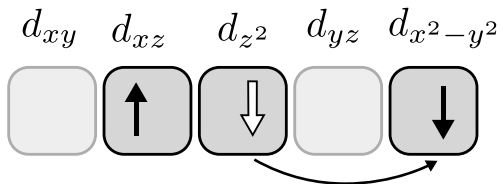

(b)

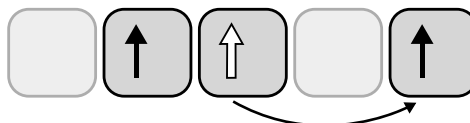

(c)

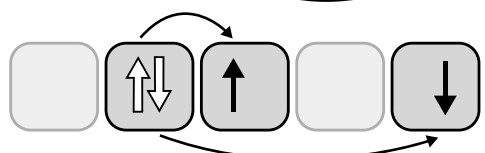

(d)

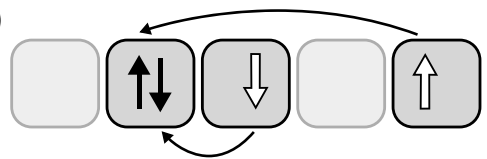

(e)

(f)
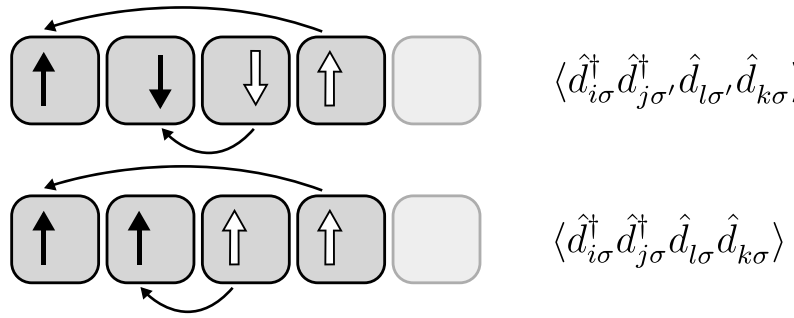

FIG. 14. Schematic representation of the three- and four-index interaction terms (excluding permutations) for the $3 d$ shell in the basis of the cubic harmonics. The white and black spins denote the initial and final configurations connected by the operator, respectively. The terms are (a), (b) correction to the Hund's exchange due to an interorbital effective hopping, (c), (d) annihilation and creation of an orbital pair, and (e), (f) two-unpaired-electrons hopping.

However, all the integrals $a_{k}$ are tabulated [29] and the numerical values of the corresponding terms can be calculated in a straightforward way for the $3 d$ shell, given the Slater integrals $F^{0}, F^{2}$, and $F^{4}$. For the values chosen here, we obtain three independent parameters in the spherical approximation, which we refer to as $J_{5}=0.18 \mathrm{eV}, J_{6}=0.35 \mathrm{eV}$, and $J_{7}=0.31 \mathrm{eV}$. These interactions appear with both positive and negative sign in the Coulomb tensor, and they are associated to three- and four-index terms, so that a complete disentangling of these contributions is a nontrivial task, even in the spherical approximation. We provide a datafile with all elements of the full Coulomb tensor $U_{i j k l}$, in the basis of the cubic harmonics, in the Supplemental Material [51].

\section{APPENDIX C: QUANTUM NUMBERS}

It is worth mentioning that all calculations for the $\mathrm{Co} / \mathrm{Cu}(001)$ system have been performed with the full Coulomb tensor, and the interaction Hamiltonian (i.e., density-density, Kanamori, or full Coulomb) is selected by requiring the conservation of a specific set of quantum numbers.

Any spin-independent two-body interaction conserves the electron number $\sum_{i \sigma} \hat{n}_{i \sigma}$ and the spin projection $\hat{S}_{z}$. The density-density interaction conserves the electron number in each spin-orbital $\hat{n}_{i \sigma}$. Instead, the Kanamori interaction conserves the quantity $\sum_{i} 2^{i}\left(\hat{n}_{i \uparrow}-\hat{n}_{i \downarrow}\right)^{2}$, which represents the pattern of orbital single occupations, also known as PS number [34], but relaxes the conservation of the electron number on each orbital, regardless of spin, by allowing the spin-flip exchange interaction term (see schematics in Fig. 13). In general, three- and four-index interaction terms included in the full Coulomb tensor conserve, e.g., neither the spin-orbital occupation nor the PS number (see schematics in Fig. 14).

In the CT-QMC calculations for the simpler interaction parametrizations using W2DYNAMICS [35], the level of simplification was specified by requiring the conservation of the appropriate quantum numbers. This causes the local state space to be partitioned in such a way that terms of the Coulomb tensor connecting states with different quantum number values do not enter into the imaginary time evolution [35].
[1] J. Kondo, Prog. Theor. Phys. 32, 37 (1964).

[2] A. C. Hewson, The Kondo Problem to Heavy Fermions (Cambridge University Press, Cambridge, UK, 1997).

[3] A. A. Abrikosov, Phys. Phys. Fiz. 2,5 (1965).

[4] H. Suhl, Phys. Rev. 138, A515 (1965).

[5] N. Knorr, M. A. Schneider, L. Diekhöner, P. Wahl, and K. Kern, Phys. Rev. Lett. 88, 096804 (2002).

[6] P. Wahl, L. Diekhöner, M. A. Schneider, L. Vitali, G. Wittich, and K. Kern, Phys. Rev. Lett. 93, 176603 (2004).

[7] N. Néel, J. Kröger, L. Limot, K. Palotas, W. A. Hofer, and R. Berndt, Phys. Rev. Lett. 98, 016801 (2007).

[8] L. Vitali, R. Ohmann, S. Stepanow, P. Gambardella, K. Tao, R. Huang, V. S. Stepanyuk, P. Bruno, and K. Kern, Phys. Rev. Lett. 101, 216802 (2008).

[9] J. Meyer, R. Ohmann, A. Nickel, C. Toher, R. Gresser, K. Leo, D. A. Ryndyk, F. Moresco, and G. Cuniberti, Phys. Rev. B 93, 155118 (2016).

[10] S. Frank and D. Jacob, Phys. Rev. B 92, 235127 (2015).
[11] H. T. Dang, M. dos Santos Dias, A. Liebsch, and S. Lounis, Phys. Rev. B 93, 115123 (2016).

[12] J. Bouaziz, F. S. M. Guimarães, and S. Lounis, arXiv:2003.01746.

[13] P. Huang and E. A. Carter, Nano Lett. 8, 1265 (2008).

[14] D. Jacob, J. Phys.: Condens. Matter 27, 245606 (2015).

[15] B. Surer, M. Troyer, P. Werner, T. O. Wehling, A. M. Läuchli, A. Wilhelm, and A. I. Lichtenstein, Phys. Rev. B 85, 085114 (2012).

[16] P. P. Baruselli, R. Requist, A. Smogunov, M. Fabrizio, and E. Tosatti, Phys. Rev. B 92, 045119 (2015).

[17] M. P. Bahlke, M. Karolak, and C. Herrmann, Phys. Rev. B 97, 035119 (2018).

[18] A. Posazhennikova and P. Coleman, Phys. Rev. Lett. 94, 036802 (2005).

[19] A. H. Nevidomskyy and P. Coleman, Phys. Rev. Lett. 103, 147205 (2009)

[20] Y. Wan, P. Phillips, and Q. Li, Phys. Rev. B 51, 14782 (1995). 
[21] W. Izumida, O. Sakai, and Y. Shimizu, J. Phys. Soc. Jpn. 67, 2444 (1998).

[22] T. Pruschke and R. Bulla, Eur. Phys. J. B 44, 217 (2005).

[23] E. Gorelov, T. O. Wehling, A. N. Rubtsov, M. I. Katsnelson, and A. I. Lichtenstein, Phys. Rev. B 80, 155132 (2009).

[24] A. I. Lichtenstein and M. I. Katsnelson, Phys. Rev. B 57, 6884 (1998).

[25] M. Schüler, S. Barthel, T. Wehling, M. Karolak, A. Valli, and G. Sangiovanni, Eur. Phys. J.: Spec. Top. 226, 2615 (2017).

[26] G. Kresse and J. Furthmüller, Phys. Rev. B 54, 11169 (1996).

[27] G. Kresse and D. Joubert, Phys. Rev. B 59, 1758 (1999).

[28] R. W. G. Wyckoff, Crystal Structures (Interscience Publishers, New York, 1963).

[29] J. C. Slater, Quantum Theory of Atomic Structure (McGrawHill, New York, 1960).

[30] E. Gull, A. J. Millis, A. I. Lichtenstein, A. N. Rubtsov, M. Troyer, and P. Werner, Rev. Mod. Phys. 83, 349 (2011).

[31] J. C. Slater, Phys. Rev. 34, 1293 (1929).

[32] A. Hausoel, M. Karolak, E. Şaşığlu, A. Lichtenstein, K. Held, A. Katanin, A. Toschi, and G. Sangiovanni, Nat. Commun. 8, 16062 (2017).

[33] M. Karolak, Ph.D. thesis, Universität Hamburg, Hamburg, Germany, 2013.

[34] N. Parragh, A. Toschi, K. Held, and G. Sangiovanni, Phys. Rev. B 86, 155158 (2012).

[35] M. Wallerberger, A. Hausoel, P. Gunacker, A. Kowalski, N. Parragh, F. Goth, K. Held, and G. Sangiovanni, Comput. Phys. Commun. 235, 388 (2019).

[36] M. T. Czyżyk and G. A. Sawatzky, Phys. Rev. B 49, 14211 (1994).
[37] V. I. Anisimov, J. Zaanen, and O. K. Andersen, Phys. Rev. B 44, 943 (1991)

[38] D.-J. Choi, P. Abufager, L. Limot, and N. Lorente, J. Chem. Phys. 146, 092309 (2017).

[39] S. Sasaki, S. De Franceschi, J. M. Elzerman, W. G. van der Wiel, M. Eto, S. Tarucha, and L. P. Kouwenhoven, Nature (London) 405, 764 (2000).

[40] S. Burdin, A. Georges, and D. R. Grempel, Phys. Rev. Lett. 85 , 1048 (2000).

[41] A. Amaricci, L. de' Medici, G. Sordi, M. J. Rozenberg, and M. Capone, Phys. Rev. B 85, 235110 (2012).

[42] K. G. Wilson, Rev. Mod. Phys. 47, 773 (1975).

[43] A. Toschi, R. Arita, P. Hansmann, G. Sangiovanni, and K. Held, Phys. Rev. B 86, 064411 (2012).

[44] L. de’ Medici, Phys. Rev. B 83, 205112 (2011).

[45] L. Fanfarillo and E. Bascones, Phys. Rev. B 92, 075136 (2015).

[46] L. Huang, T. O. Wehling, and P. Werner, Phys. Rev. B 89 , 245104 (2014).

[47] Š. Pick, V. S. Stepanyuk, A. N. Baranov, W. Hergert, and P. Bruno, Phys. Rev. B 68, 104410 (2003).

[48] www.gauss-centre.eu.

[49] www.lrz.de.

[50] A. V. Chubukov and D. L. Maslov, Phys. Rev. B 86, 155136 (2012).

[51] See Supplemental Material at http://link.aps.org/supplemental/ 10.1103/PhysRevResearch.2.033432 for the full Coulomb tensor in the basis of cubic harmonics, and the $\mathrm{Co} / \mathrm{Cu}(001)$ VASP input file.

[52] I. Schnell, G. Czycholl, and R. C. Albers, Phys. Rev. B 68, 245102 (2003). 